\title{
A uniform CO survey of the molecular clouds in Orion and Monoceros
}

\author{
B. A. Wilson ${ }^{1,2}$, T. M. Dame ${ }^{2}$, M. R. W. Masheder ${ }^{1}$, and P. Thaddeus ${ }^{2}$ \\ ${ }^{1}$ University of Bristol, Tyndall Avenue, Bristol, UK \\ ${ }^{2}$ Harvard-Smithsonian Center for Astrophysics, 60 Garden Street, Cambridge, MA 02138, USA \\ e-mail: [awilson; tmdame]@cfa.harvard.edu
}

Received 23 December 2003 / Accepted 30 August 2004

\begin{abstract}
We report the results of a new large scale survey of the Orion-Monoceros complex of molecular clouds made in the $J=1 \rightarrow 0$ line of ${ }^{12} \mathrm{CO}$ with the Harvard-Smithsonian $1.2 \mathrm{~m}$ millimetre-wave telescope. The survey consists of 52288 uniformly spaced spectra that cover an area of $432 \mathrm{deg}^{2}$ on the sky and represent the most sensitive large-scale survey of the region to date. Distances to the constituent molecular clouds of the complex, estimated from an analysis of foreground and background stars, have provided information on the three dimensional structure of the entire complex.
\end{abstract}

Key words. ISM: clouds - ISM: structure - ISM: kinematics and dynamics - ISM: general

\section{Introduction}

Orion-Monoceros is one of the most important and best studied regions of star-formation. Much of its importance derives from its favourable position on the sky and its proximity: it is located $\sim 15^{\circ}$ below the Galactic plane and toward the outer Galaxy well away from the potentially confusing molecular gas of the plane and the Galactic centre; at a distance of $\sim 450 \mathrm{pc}$, it is relatively close, and its constituent clouds can be observed with good linear resolution even with a radio telescope of modest size. The radial velocities, proper motions and parallaxes of the stars associated with the molecular gas are observable, and therefore the distances to the clouds and the three-dimensional structure of the complex can be estimated.

Orion-Monoceros provides an excellent laboratory for studying the interaction between massive stars and the interstellar medium (ISM). Over the course of the last $12 \mathrm{Myr}$ the molecular clouds in the region have been shaped, compressed and disrupted by the powerful ionizing radiation, stellar winds and supernova ( $\mathrm{SN}$ ) explosions of the young massive stars in the Orion OB association (Cowie et al. 1979; Bally et al. 1987). Compression of the clouds may have triggered bursts of starformation (Elmegreen \& Lada 1977) that are traced by the four semi-distinct Ori OB1 subgroups. The oldest subgroup, OB $1 \mathrm{a}$, lies in a region about $10^{\circ}$ from the main clouds longago cleared of molecular gas, while the youngest $\mathrm{OB} 1 \mathrm{~d}$ is currently forming the stars in the Orion nebulae on the near side of the Orion A cloud. As well as disrupting the molecular gas in Orion-Monoceros, the stars of Ori OB1 have also inflated a huge elongated bubble of hot X-ray emitting gas, the Orion-Eridanus supershell (Heiles et al. 1999), which is centered on the Orion star-forming region $15^{\circ}-20^{\circ}(\sim 150 \mathrm{pc})$ below the Galactic Plane. As the bubble has expanded it has swept up a massive shell of dust and gas that has become an optically bright crescent (Barnard's Loop) as it moved into the steep density and pressure gradients associated with the Galactic gas layer. Figure 1 shows a finder diagram for OrionMonoceros. The greyscale ${ }^{1}$ represents the integrated $\mathrm{H} \alpha$ emission as measured by the Wisconsin $\mathrm{H} \alpha$ mapper (WHAM Reynolds et al. 1998), and the contours show the boundaries of the main molecular clouds as traced by the $\mathrm{CO}$ survey presented here. The positions of the $\mathrm{O}$ and $\mathrm{B}$ stars from the four Ori-OB 1 subgroups, from Brown et al. (1995), are also plotted.

The first observations of molecular emission from OrionMonoceros, which were also the first detections of interstellar CO, were made toward the Orion nebula by Wilson et al. (1970) using the $J=1 \rightarrow 0$ line of the normal isotopic species. Subsequent studies using this line by Kutner et al. (1977), Chin (1977), and in particular Maddalena et al. (1986) were conducted primarily to determine the spatial extent of the complex. It was found that the complex contains three giant $\left(10^{5} M_{\odot}\right)$ molecular clouds (GMCs), Orion A, Orion B and Mon R2, two long filaments which extend approximately $10^{\circ}$ from the cloud complex to the Galactic plane, and numerous smaller clouds. Parts of the complex have subsequently been observed in $\mathrm{CO}$ at higher resolution either using the $J=1 \rightarrow 0$ line or other isotopes or transitions. Most importantly, the majority of Mon R2 was mapped in CO $(J=1 \rightarrow 0)$ by Xie \& Goldsmith (1994), the central part of Orion A was mapped using ${ }^{13} \mathrm{CO}$ $(J=1 \rightarrow 0)$ by Bally et al. (1987), by Nagahama et al. (1998), and in the $J=1 \rightarrow 0$ and $J=2 \rightarrow 1$ lines of ${ }^{12} \mathrm{CO},{ }^{13} \mathrm{CO}$ and $\mathrm{C}^{18} \mathrm{O}$ by Castets et al. (1990). In addition, the southern

\footnotetext{
1 The electronic version of this paper contains colour figures.
} 


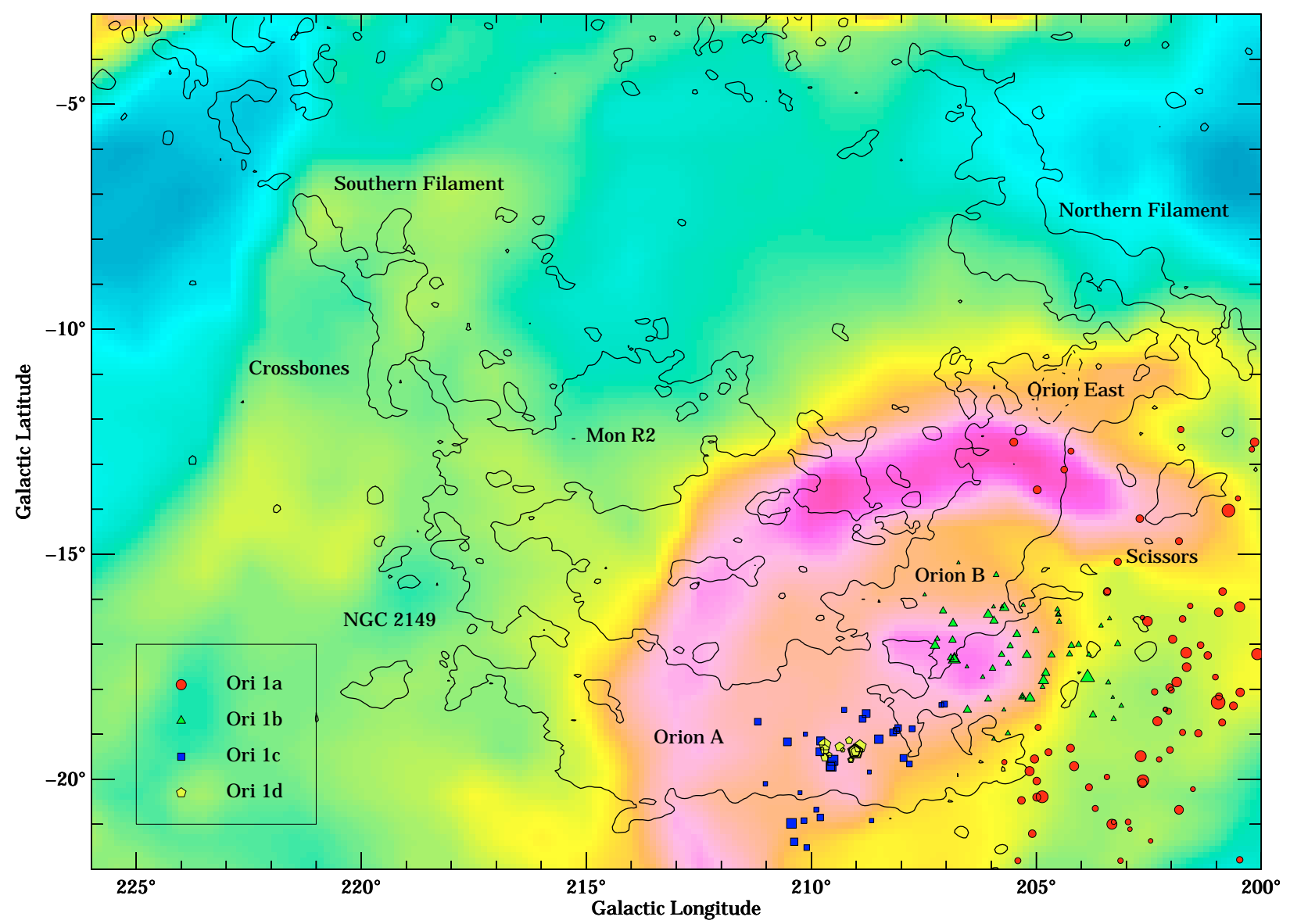

Fig. 1. Finder diagram for Orion-Monoceros. The greyscale represents the integrated intensity of ionized gas as traced by the WHAM H $\alpha$ survey (Reynolds et al. 1998). The bright crescent of emission is Barnard's loop which delineates the optical boundary of the Orion-Eridanus superbubble. The positions of O and B stars from the Ori OB1 association (Brown et al. 1995) are plotted with symbols the sizes of which are proportional to the luminosity of the stars. The four different symbols correspond to the four Ori OB 1 subgroups. The boundary of the Orion-Monoceros molecular complex is marked by the $2.06 \mathrm{~K} \mathrm{~km} \mathrm{~s}^{-1}(3 \sigma)$ contour of the new CO survey.

part of Orion B was mapped in $\mathrm{CO}$ and ${ }^{13} \mathrm{CO}(J=2 \rightarrow 1$ and $J=3 \rightarrow 2$ ) by Kramer et al. (1996), and the Orion A and B clouds were mapped using the $J=2 \rightarrow 1$ line of $\mathrm{CO}$ (Sakamoto et al. 1994). However, to date the only survey which has covered the whole region has been that of Maddalena et al. (1986), a survey which is non-uniform in resolution, sensitivity and sampling, and is heavily undersampled in many places.

This paper presents a new CO $(J=1 \rightarrow 0)$ survey of the Orion-Monoceros complex which consists of 52288 spectra uniformly spaced in Galactic longitude and latitude. It is generally four times more sensitive than the Maddalena et al. (1986) survey, since it contains four times as many spectra, each with lower rms noise. The new observations cover a total area of more than $400 \mathrm{sq}$ deg on the sky and map all of the major clouds of the Orion-Monoceros complex with the exception of the $\lambda$ Orionis ring, which was the subject of a separate study with the $1.2 \mathrm{~m}$ telescope (Lang et al. 2000).

This paper is organised as follows: in Sect. 2 the observations and data reduction procedure are described. In Sect. 3 the methods used to estimate the masses of and the distances to specific regions in the Orion-Monoceros complex are outlined. In Sect. 4 these specific regions are described in detail. Section 5 presents an overview of the large scale structure of the complex and describes a possible formation scenario. A summary and conclusions are presented in Sect. 6 .

\section{Observations}

The $1.2 \mathrm{~m}$ millimetre wave telescope at the HarvardSmithsonian Center for Astrophysics was used for all the $J=1 \rightarrow 0{ }^{12} \mathrm{CO}$ observations presented here. This telescope has a beamwidth $(F W H M)$ of 8.4 and a velocity resolution of $0.65 \mathrm{~km}^{-1}$ at $115.2712 \mathrm{GHz}$, provided by a 256 channel filter bank. Spectral line intensities were calibrated against an ambient temperature blackbody, a standard Eccosorb chopper wheel (Davis et al. 1973), which was rotated in front of the feed horn at $20 \mathrm{~Hz}$ for $1 \mathrm{~s}$ before each observation. All line intensities reported here are in units of main beam brightness temperature $T_{\mathrm{mb}}=T_{\mathrm{A}}^{*} / \eta$, where $T_{\mathrm{A}}^{*}$ is the chopper calibrated antenna temperature (Penzias et al. 1973) (i.e. the antenna temperature corrected for atmospheric attenuation, ohmic losses and rearward spillover), and $\eta$ is the main beam efficiency, 0.82 . The emission in Orion is so strong and extensive that $T_{\mathrm{mb}}$ will at some locations overestimate the intensity in the main beam by a factor up to $5 \%$ owing to emission in the near sidelobes. 


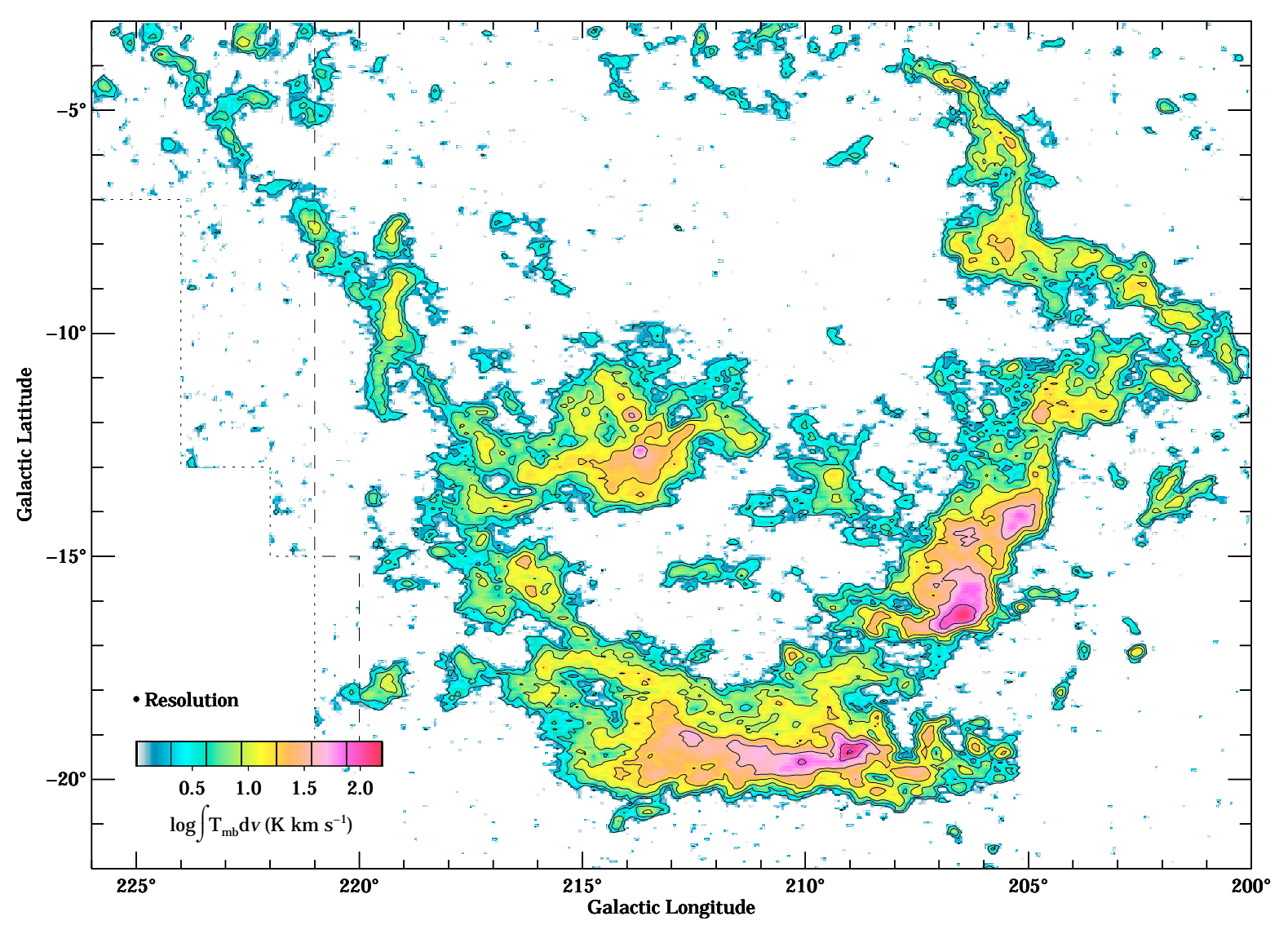

Fig. 2. Log velocity-integrated $(J=1 \rightarrow 0)$ CO map of Orion-Monoceros. The integration range is $-3.0<v_{\mathrm{LSR}}<19.5 \mathrm{~km} \mathrm{~s}{ }^{-1}$. Contours start at $0.313(3 \sigma)$ and go up in steps of $0.313 \log \left(\mathrm{K} \mathrm{km} \mathrm{s}^{-1}\right)$. The dashed line marks the limit of the full-resolution survey. The region on the high longitude side of the dashed line has been sampled with beamwidth spaced observations; that on the low longitude side has been observed twice with the second set of observations offset by half a beamwidth in longitude and latitude.

The detector was a liquid-helium cooled SIS receiver with a single sideband noise temperature of $\sim 75 \mathrm{~K}$ (Pan 1983).

Measurements of the sky brightness as a function of elevation were obtained by antenna tipping at least every six hours and more frequently during periods of changeable weather, and were fitted to the two layer atmospheric model of Kutner (1978) to determine the temperature and opacity of atmospheric water vapour. Further details on the receivers, observational technique and calibration of the $1.2 \mathrm{~m}$ telescope can be found in Dame et al. (1993). During the course of the observations total system temperatures referred to above the atmosphere ranged from $\sim 900 \mathrm{~K}$ at the lowest elevations observed $\left(30^{\circ}\right)$ to $\sim 500 \mathrm{~K}$ at the highest $\left(\sim 60^{\circ}\right)$. Integration times were automatically adjusted to achieve an rms noise temperature per channel of $\sim 0.26 \mathrm{~K}$.

Frequency switched observations were carried out over four consecutive observing seasons from February 1999 to April 2002 , using two 0.125 grids that were offset by 0.0625 in Galactic Longitude and Latitude. The beam spacing of the final composite grid is 0.09 , and the $x$ and $y$ axes of the grid are tilted at $45^{\circ}$ with respect to $l$ and $b$. The frequency switched spectra were folded and 5th order polynomial baselines fitted to the portion of each spectrum that was found to be free of emission. For each observing session of $\sim 6 \mathrm{~h}$ a model of the $\mathrm{CO}$ emission feature associated with the mesospheric layer of the Earth's
Table 1. Parameters of the survey.

\begin{tabular}{ll}
\hline \hline Number of spectra & 52288 \\
Galactic longitude & $200^{\circ} \rightarrow 226^{\circ}$ \\
Galactic latitude & $-22^{\circ} \rightarrow-3^{\circ}$ \\
Angular resolution & 8.4 \\
Sampling interval & 0.088 \\
Velocity resolution & $0.65 \mathrm{~km} \mathrm{~s}^{-1}$ \\
Sensitivity & $T_{\mathrm{mb}}=0.26 \mathrm{~K} \mathrm{rms}$ \\
Frequency switching interval & $15 \mathrm{MHz}$ \\
\hline
\end{tabular}

atmosphere was fitted to all spectra that were free of Galactic CO emission (Lang 1997). This model was then used to remove the mesospheric CO line from all the spectra taken for that session. Finally the oversampled observations were smoothed by convolution with a two-dimensional Gaussian the size of the telescope's main beam in order to lower the final rms noise to $\sim 0.14 \mathrm{~K}$. The parameters of the pre-smoothed observations are summarized in Table 1.

The CO observations are summarized in Fig. 2, which shows the spatial distribution of $W_{\mathrm{CO}}\left(\mathrm{K} \mathrm{km} \mathrm{s}^{-1}\right)$, the velocityintegrated line intensity. The $v_{\mathrm{LSR}}$ integration interval of -3 to $19.5 \mathrm{~km} \mathrm{~s}^{-1}$ was chosen after an examination of the channel maps in order to include all the regions in the spectra where signal was present. The higher sensitivity per unit area 

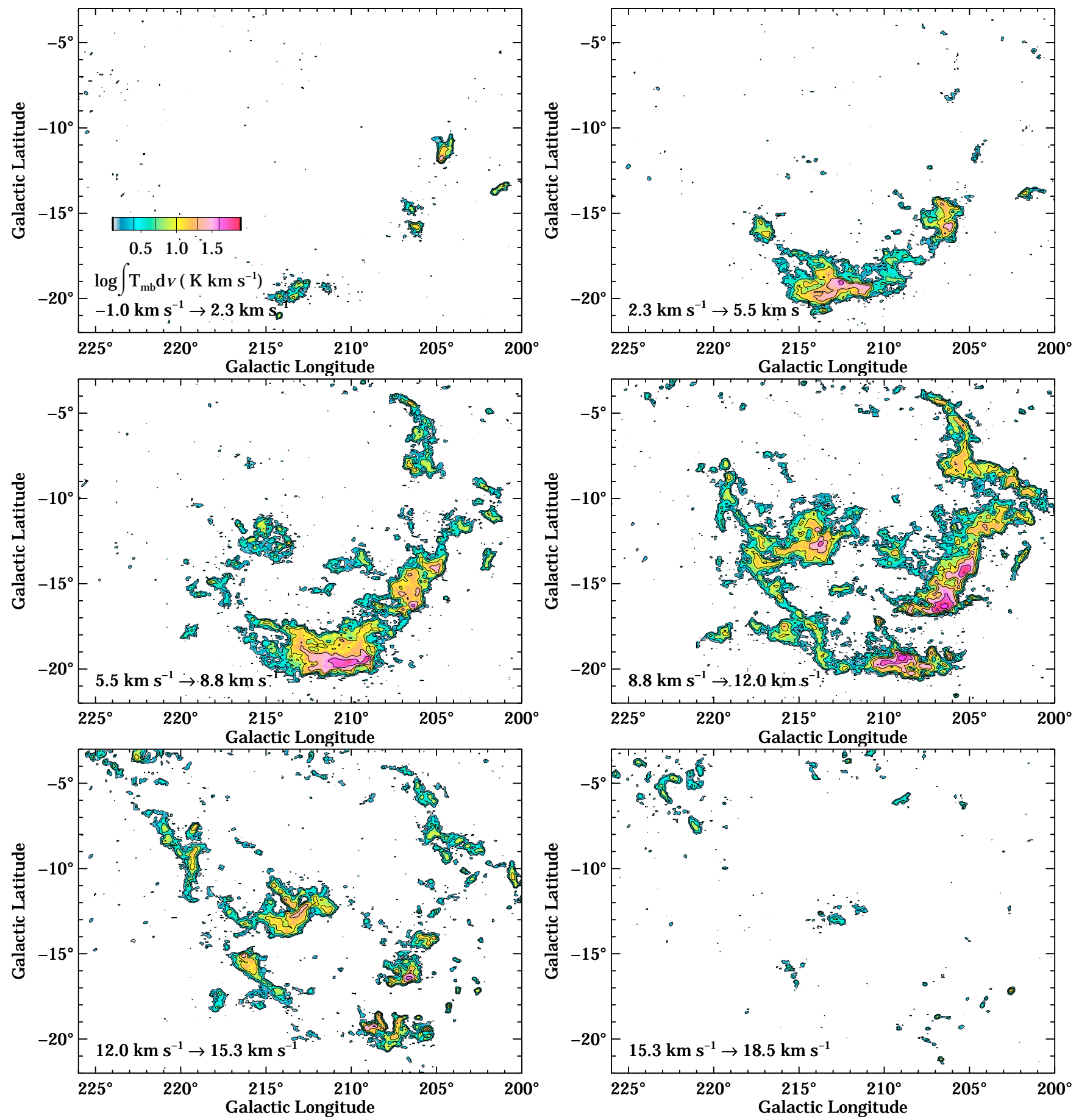

Fig. 3. Channels maps. Each map shows the integrated emission from five velocity channels (total width $3.25 \mathrm{~km} \mathrm{~s}^{-1}$ ) from -1.0 to $18.5 \mathrm{~km} \mathrm{~s}^{-1}$. Log contour levels start at $0.1(3 \sigma)$ and go up in steps of 0.3 .

of the new survey is apparent in the amount of emission that is now detected from the areas between the main molecular clouds. Although weak, this emission bridges the clouds spatially and without discontinuity in velocity, indicating that none of the major clouds are entirely isolated. The velocity structure of the region is shown in the channel maps, Fig. 3, and the emission-weighted mean velocity map Fig. 4. These two figures indicate that the majority of the emission is confined to the relatively narrow velocity range $2 \rightarrow 15 \mathrm{~km} \mathrm{~s}^{-1}$; although individual clouds such as Orion A and the Southern Filament have systematic velocity gradients, there is none for the complex as a whole. The structure and kinematics of the individual regions within the complex are discussed in detail in Sect. 4.

\section{Molecular masses and cloud distances}

In spite of clear evidence of saturation in the denser cores of molecular clouds, the ${ }^{12} \mathrm{CO}$ line remains the best overall tracer of mass in giant molecular clouds, since most of the mass is contained in the lower-density envelopes (Cambresy 1999). 


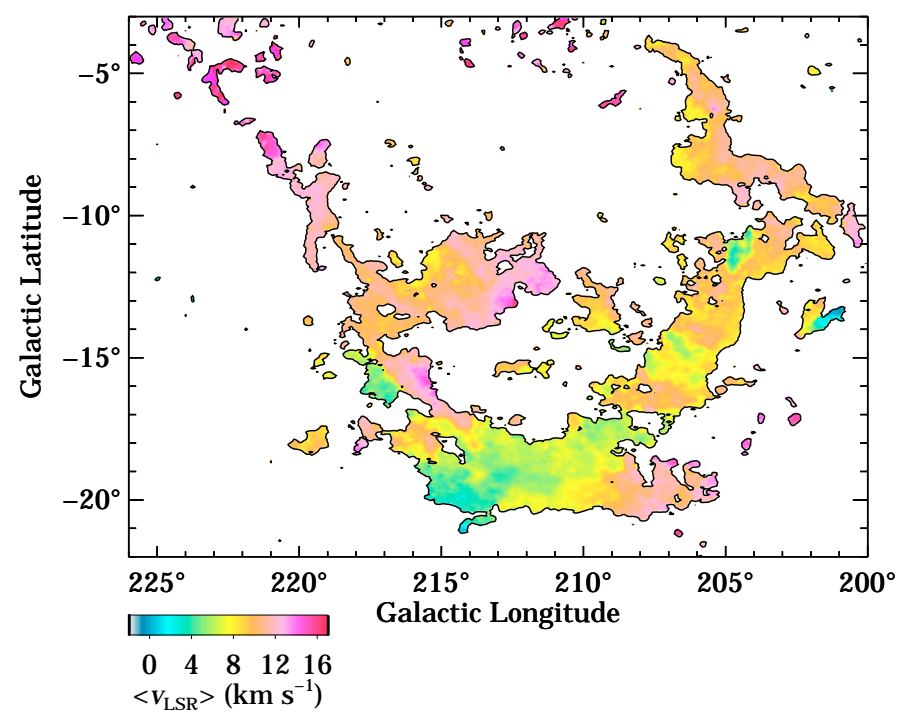

Fig. 4. Emission weighted mean velocity map of Orion-Monoceros. This figure clearly shows the velocity gradients along Orion A and the Southern Filament and highlights regions with unusual kinematics such as the Scissors and NGC 2149.

Cloud masses in the Orion-Monoceros complex were estimated using the standard assumption of a linear relationship between the velocity integrated $\mathrm{CO}$ intensity, $W_{\mathrm{CO}}$, and the molecular hydrogen column density, $N\left(\mathrm{H}_{2}\right)$, taking

$N\left(\mathrm{H}_{2}\right) / W_{\mathrm{CO}}=(1.8 \pm 0.3) \times 10^{20} \mathrm{~cm}^{-2} \mathrm{~K}^{-1} \mathrm{~km}^{-1} \mathrm{~s}^{-1}$

as derived by Dame et al. (2001). This yields

$M_{\mathrm{CO}} / M_{\odot}=1200 S_{\mathrm{CO}} d_{\mathrm{kpc}}^{2}$

where $d$ is the distance to the cloud in kpc, and $S_{\mathrm{CO}}$ is the CO emission integrated over velocity and the angular extent of the cloud in $\mathrm{K} \mathrm{km} \mathrm{s}^{-1}$ arcdegrees ${ }^{2}$. A mean atomic weight of 1.36 is assumed in order to account for helium and heavier elements (Allen 1973).

Historically the distances to Orion A and B have been assumed to be the same as the distance to the Orion nebula which has been estimated photometrically as $435 \mathrm{pc}$ (Warren et al. 1977 ), and as $480 \pm 80 \mathrm{pc}$ via a study of the radial velocities and proper motions of masers in the OMC 1 core (Genzel et al. $1981)^{2}$. Although these estimates may be accurate, the implicit assumption that all the clouds are at the same distance is unsatisfactory. At $450 \mathrm{pc}$ the Orion A cloud would be over $100 \mathrm{pc}$ long, and although the region near to the Orion nebula is likely to be at a distance close to the photometric estimate, the other end of the cloud may be significantly nearer or further away.

In order to address this problem the parallaxes of stars observed with the astrometric satellite Hipparcos have been used to estimate distances to the molecular clouds in OrionMonoceros. Individual stars are identified as being in front or behind a particular molecular cloud by comparing its colour with two theoretical models: the colour of the star if it is reddened by dust associated with atomic and molecular gas (i.e. it

${ }^{2}$ Mon R2 is generally believed to be further away at $830 \pm 50 \mathrm{pc}$ (Racine 1968; Herbst \& Racine 1976) and not directly related to the other clouds in the complex. is in the background of the cloud), and the reddening associated with just atomic gas (i.e. it is in the foreground of the cloud). The parallaxes of the stars that can be assigned unambiguously to the foreground or the background of a molecular cloud are then used to constrain the distance to that cloud.

In general Hipparcos parallaxes for single stars are unreliable for distances greater than $\sim 200 \mathrm{pc}$, but by using groups of foreground and background stars and carefully modelling the probability distribution function ( $\mathrm{pdf}$ ) for the cloud distance it is possible to make robust distance estimates out to approximately $500 \mathrm{pc}$. Furthermore, in some cases it has been possible to divide the larger clouds into multiple sections and make separate distance estimates for each part. In these cases the velocity structure of the complex was used as a guide when subdividing a cloud.

Table 2 lists the distances and masses of the various clouds in Orion-Monoceros estimated from Hipparcos parallaxes. Most of these distances agree, within the errors, with previous estimates made using other techniques. The distance determination technique is described in more detail together with specific examples for Orion and other local molecular clouds in Wilson et al. (2002).

\section{Specific regions}

\subsection{Orion $A$}

The Orion A cloud, the largest in the Orion-Monoceros complex, is located $\sim 19^{\circ}$ below the Galactic plane at a distance of $\sim 450 \mathrm{pc}$ from the Sun. It has a surface area of $\sim 31.5 \mathrm{deg}^{2}$ $\left(\sim 2200 \mathrm{pc}^{2}\right)$ and if cylindrical in shape, has a depth of $\sim 20 \mathrm{pc}$. Figure 5a shows the velocity-integrated $\mathrm{CO}$ emission from Orion A which is somewhat cometary in appearance, particularly above the $24 \mathrm{~K} \mathrm{~km} \mathrm{~s}^{-1}$ contour. The apparent head of the comet is the compact knot of emission at $l=209^{\circ}$ and $b=-19.25$, and the ridge of dense gas that points away from this peak (and also away from the centroid of the OB association), becomes a wider lower density tail toward higher longitudes.

The longitude-velocity map in Fig. $5 \mathrm{~b}$ shows the large-scale velocity gradient that runs along the length of the cloud. First reported by Kutner et al. (1977), this gradient has been confirmed by all subsequent large-scale observations of the cloud. Its origin has been attributed to both rotation (Kutner et al. 1977; Maddalena et al. 1986) and to large scale expansion driven by the stellar winds of the Ori OB1 association (Bally 1987). In the first case the rotation is about an axis perpendicular to the Galactic plane and opposed to the rotation of the Galaxy. Heyer et al. (1992) argue that this model is unlikely to be correct as the velocity-gradient (as shown by their data) is not sufficiently continuous over the length of the major axis to be consistent with the cloud's rotational motion. Specifically, there exist sharp velocity shifts of $\sim 1 \mathrm{~km} \mathrm{~s}^{-1}$ between adjacent spectra at many locations. Bally et al. (1987) and Heyer et al. (1992) also identify many regions within Orion A that exhibit very sharp emission gradients and velocity shifts which they attribute to expanding shells driven by HII regions excited by young massive stars. However, the velocity 
Table 2. Masses and distances for the molecular clouds in the Orion-Monoceros complex.

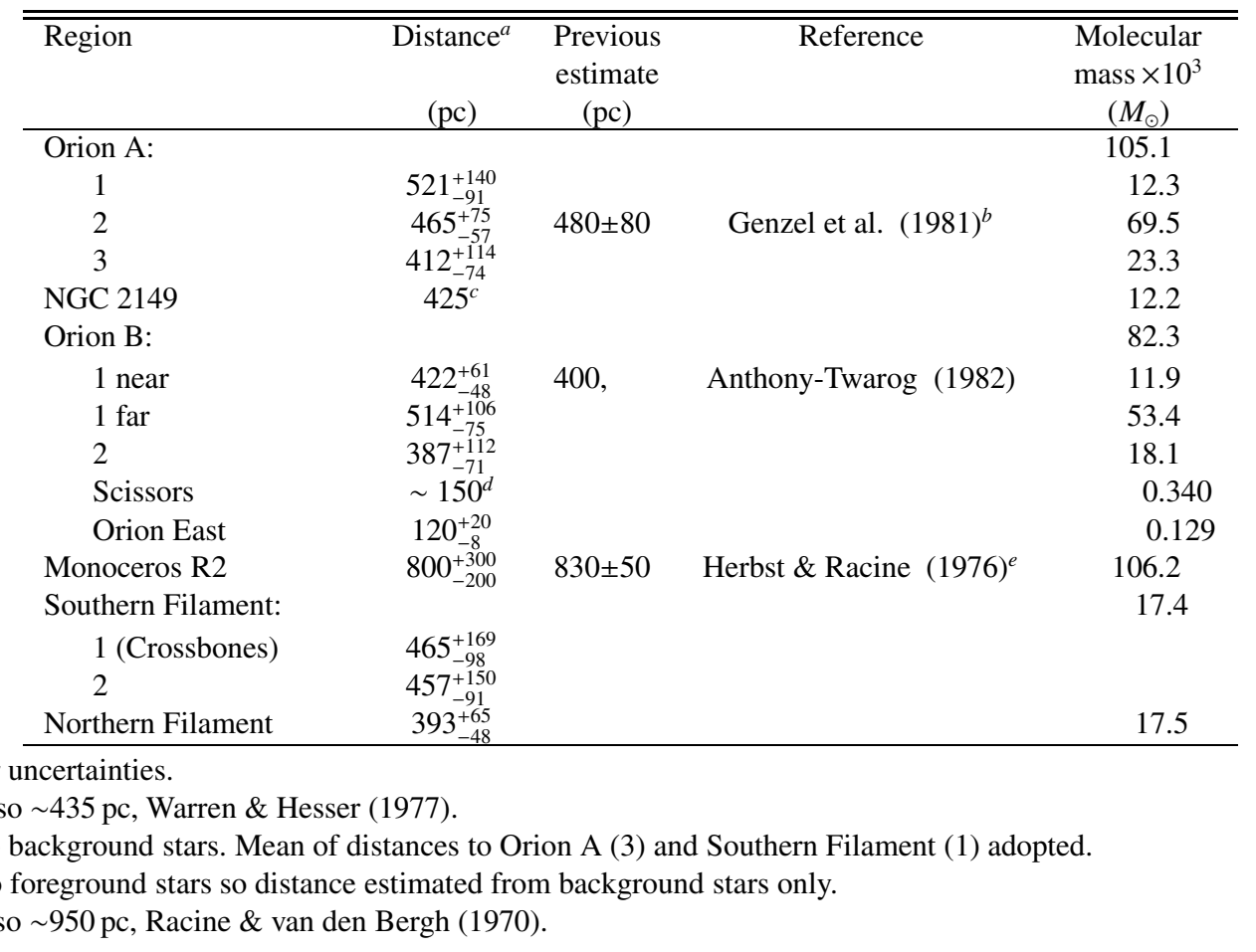

gradient as shown in Fig. 5b extends over a distance of $\sim 100 \mathrm{pc}$ and it is unlikely that such a coherent structure is the result of purely internal processes. It is possible, however, that the gradient was formed by the passage through the cloud of a much larger shell, similar to those driven by the Ori OB association. It is noted that it is not possible to see the huge Orion A outflow in the longitude-velocity map (Fig. 5b) as the data has been integrated over $5^{\circ}$. However, the Orion A outflow can be seen clearly in narrower position-velocity slices (see Sect. 5).

Distances to Orion A were determined for three sections; delineated by the vertical dotted lines in Fig. 5a at $208^{\circ}$ and $213^{\circ}$, using the Hipparcos constraining star method described in Sect. 3. The distances were estimated to be $520 \mathrm{pc}, 465 \mathrm{pc}$ and 410 pc for Regions 1, 2 and 3 respectively. Although adjacent distance estimates are roughly equal (cf. Table 2), they imply that the cloud is oriented in a direction consistent with the rotation model of Kutner (1977) and Maddelena (1986). However, this model is unable to explain the velocity jump between Regions 1 and 2. A more likely explanation for the different distances is suggested by the locations of the stars of the OB 1 b subgroup (cf. Fig. 1) which are believed to be located at a distance of $\sim 360 \mathrm{pc}$ (Brown et al. 1994) in front of the low-longitude end of the cloud. A model where Region 1 is accelerated away from the Sun by the energy released from the massive stars of OB $1 \mathrm{~b}$ is entirely consistent with the kinematics of the cloud. Furthermore there are several small clouds (for example near $l=202^{\circ} .5, b=-17^{\circ}$ ) located toward the OB $1 \mathrm{~b}$ association that also have high velocities consistent with this model.

If the low-longitude end of Orion A has been accelerated away from the Sun by the stellar winds of the OB 1b subgroup then we would expect to see a corresponding lateral acceleration of the gas away from the centre of the subgroup. The $-1.0 \rightarrow 8.8 \mathrm{~km} \mathrm{~s}^{-1}$ channel maps (Fig. 3) show that the area around $l \approx 205^{\circ}, b \approx-19^{\circ}$ (the part of the cloud closest to the OB $1 \mathrm{~b}$ subgroup) has been cleared of molecular gas at low velocities $\left(\leq 5 \mathrm{~km} \mathrm{~s}^{-1}\right)$. In this velocity range the gas in Orion $\mathrm{A}$, and on the low-latitude side of Orion B, may also have been compressed laterally, a process that has given rise to the pronounced step in the Orion A longitude-velocity map at $\sim 208^{\circ} .5$. The velocity step is coincident with both the peak CO emission and the position of what is presently the most active site of starformation within the complex, the Orion nebula (Hillenbrand 1997). The fact that this region of high density gas (at $\sim 465 \mathrm{pc}$ ) and the young stars of the Orion Nebula (at $\sim 435 \mathrm{pc}$; Warren \& Hesser 1977) appear to be at similar distances is not entirely unexpected. It is difficult to avoid the conclusion that the OB $1 \mathrm{~b}$ subgroup compressed the gas in the proto-Orion A cloud which triggered the formation of the OB 1c and OB 1d associations.

Moving along Orion A away from the centre of the OB1c subgroup and toward higher longitudes, the gas becomes more diffuse and there is a marked decrease in star-formation. Region 3 of the Orion A cloud, the area between $l \approx 213^{\circ}$ and $l \approx 217^{\circ}$, contains two components that are distinct both in position and velocity. Maddalena et al. (1986) suggested that the second, higher velocity component belonged to a separate group of clouds associated with NGC 2149 that is not directly related to Orion A. It will be shown in section 4.5 that the NGC 2149 clouds in fact form a coherent structure with the high longitude end of the Orion A cloud and part of the Southern Filament. 


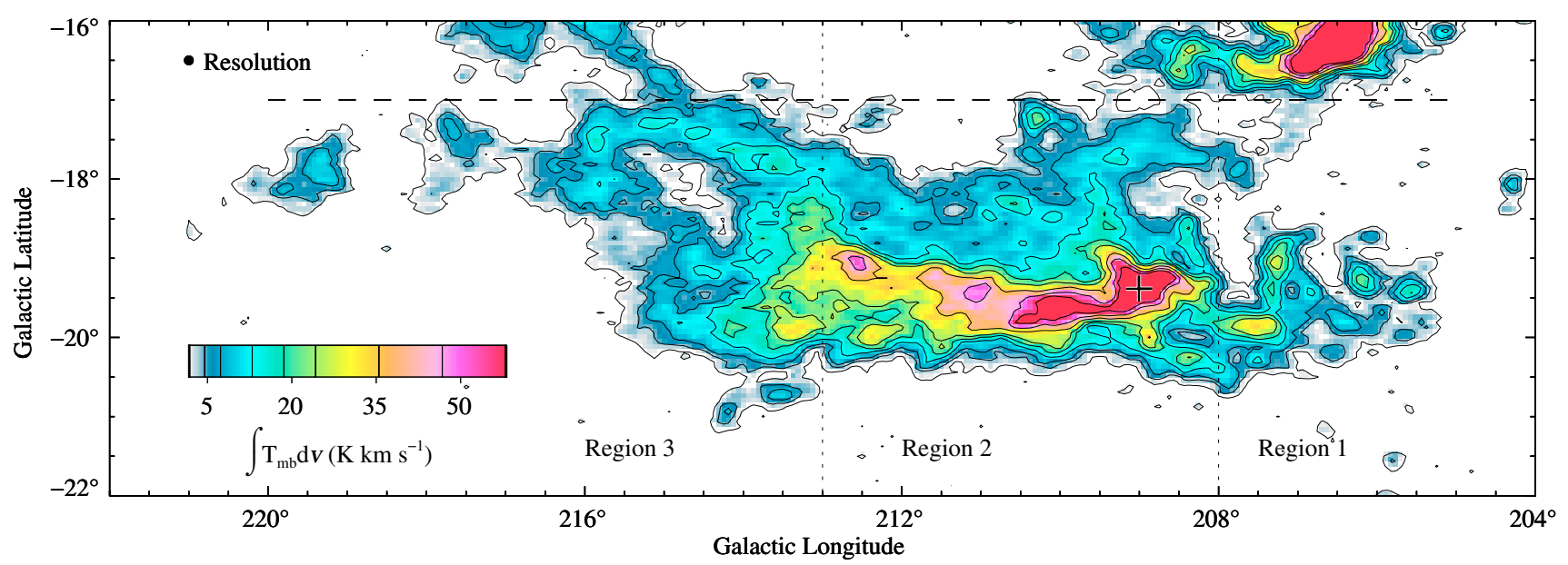

(a)

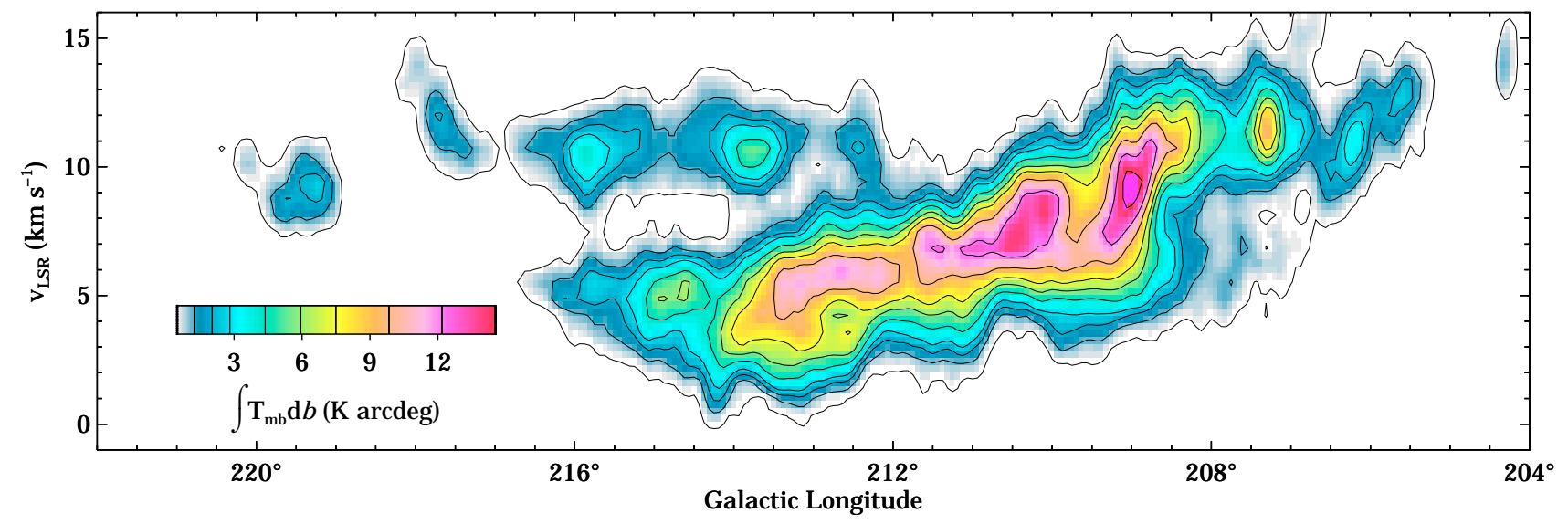

(b)

Fig. 5. a) $W_{\mathrm{CO}}$ map of Orion A. The integration range is $0.0<v_{\mathrm{LSR}}<15.0 \mathrm{~km} \mathrm{~s}^{-1}$. Contours start at $1.68 \mathrm{~K} \mathrm{~km} \mathrm{~s}^{-1}(3 \sigma)$ and subsequent levels are at $8,13,23,33,43,63,83$ and $103 \sigma$. The dashed horizontal line marks the nominal boundary between Orion A and Orion B, the dotted vertical lines separate the three regions for which distances were estimated and the cross marks the position of the Orion Nebula. b) Longitudevelocity map of Orion A. The integration range is $-22^{\circ}<b<-17^{\circ}$ and the contours start at $0.468 \mathrm{~K} \operatorname{arcdeg}(3 \sigma)$ with subsequent levels at 3 , $8,13,18,28,38,48,63,78$ and $93 \sigma$.

\subsection{Orion $B$}

Orion B is the second largest cloud in the Orion-Monoceros complex, at least in apparent size. The cloud subtends an area of $\sim 25.7 \mathrm{deg}^{2}$, which at $450 \mathrm{pc}$ is $1500 \mathrm{pc}^{2}$, and it has a mass of $0.8 \times 10^{5} M_{\odot}$. The $\mathrm{CO}$ emission from Orion B is summarized in Fig. 6, which shows (a) the $W_{\mathrm{CO}}$ map and (b) the latitudevelocity map. The dashed line at $b=-17^{\circ}$ on the $W_{\mathrm{CO}}$ map marks the nominal boundary between the Orion B and Orion A clouds. Emission from the "Scissors" (discussed below) was not included in the latitude-velocity map.

The emission from Orion B peaks at $l=206.5, b=-16.5$, toward the HII region NGC 2024 and the reflection nebula NGC 2023. A ridge of high density gas extends from the CO peak along the very well defined low-latitude edge of the cloud. The low-longitude side of the cloud, the side closest to the centres of the OB 1a and OB 1b subgroups, also shows a sharp edge in $\mathrm{CO}$ emission. These sharp edges suggest that the boundary between the molecular and the atomic gas is seen close to edge on. By contrast there is a network of increasingly diffuse ridges and filaments that trail off toward the opposite side of the cloud, in directions that point away from the centre of the OB association.

It can be seen from the velocity-latitude plot in Fig. $6 \mathrm{~b}$ that the gas in Orion B does not possess a systematic velocity gradient and that the cloud as a whole is centered at $v \approx 10 \mathrm{~km} \mathrm{~s}^{-1}$. However, the majority of spectra from the region below $b=-14^{\circ}$ contain additional emission at a lower velocity $\left(v \approx 7 \mathrm{~km} \mathrm{~s}^{-1}\right)$. This excess can also be seen in the low-velocity channel maps (Fig. 3). Many of the CO lines from this region are either double peaked or unusually wide and lopsided with the excess at low velocities. Toward higher Galactic latitudes, around $l=204^{\circ} .5, b=-11^{\circ} .5$, a second cloud is superimposed upon Orion B. Associated with the dark nebulae L 1621 and L 1622 (Lynds 1962) and designated Orion East by Maddalena et al. (1986), this cloud is at a velocity of $\sim 1 \mathrm{~km} \mathrm{~s}^{-1}$ and may have little connection to the main Orion B cloud, though it seems kinematically related to the Orion-Eridanus superbubble. Orion East is discussed in the next section.

Orion B was divided into two sections, and distances estimated for each. The area located below $b=-14^{\circ}$ which 


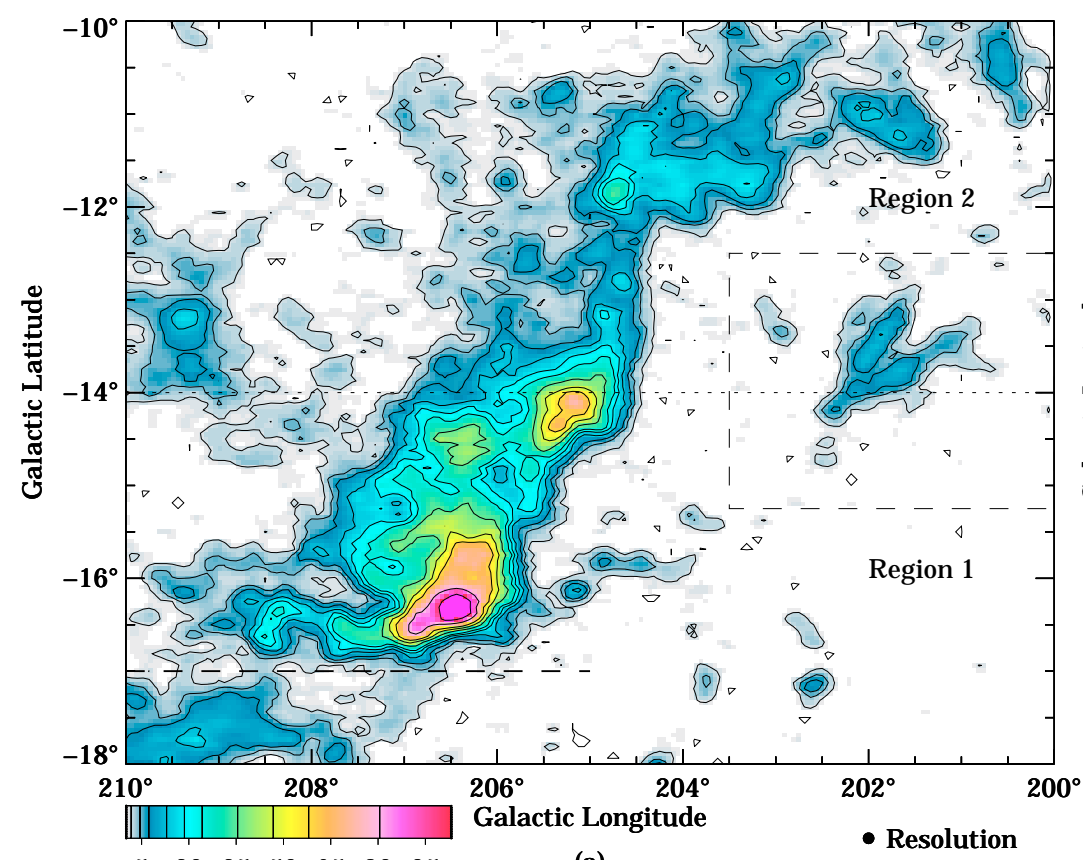

5203550658095 $\int \mathrm{T}_{\mathrm{mb}} \mathrm{d} v\left(\mathrm{~K} \mathrm{~km} \mathrm{~s}^{-1}\right)$

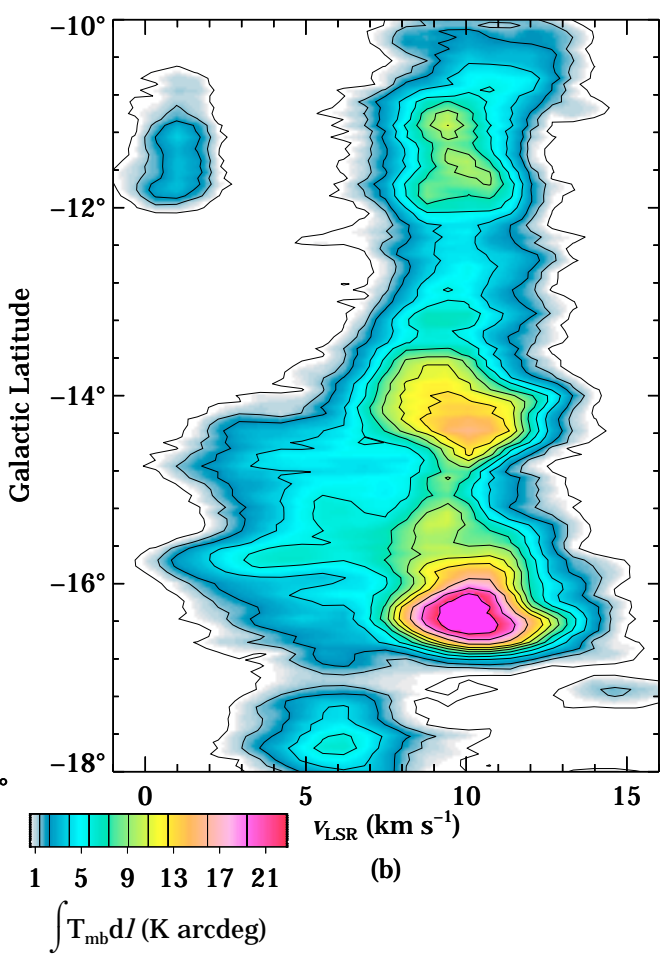

Fig. 6. a) $W_{\mathrm{CO}}$ map of Orion B. The integration range is $0<v_{\mathrm{LSR}}<15 \mathrm{~km} \mathrm{~s}^{-1}$. Contours start at $1.68 \mathrm{~K} \mathrm{~km} \mathrm{~s}^{-1}(3 \sigma)$ and subsequent levels are at $8,13,23,33,43,63,83,103,143$ and $183 \sigma$. The horizontal dashed line at $b=-17^{\circ}$ marks the nominal boundary between the Orion A and Orion B clouds. The horizontal dotted line at $b=-14^{\circ}$ separates the clouds into two regions for which distances were estimated. Emission from "the Scissors", marked by the dashed box was not included in the velocity-latitude map. b) Velocity-latitude map of Orion B. The integration range is $200^{\circ}<l<210^{\circ}$ and the contours start at $0.514 \mathrm{~K}$ arcdeg $(3 \sigma)$ with subsequent levels at $8,13,23,33,43,53,63,73,93,113$ and $133 \sigma$.

contains the CO peaks associated with NGC 2023, NGC 2024, NGC 2068 and NGC 2071 was designated Region 1. The region of more diffuse gas above $b=-14^{\circ}$ was designated Region 2. When the foreground-background assignment was made for Region 1 there were three stars whose extinction was four times below the value that would be expected if they were located behind the cloud, but $\sim 3$ times above the extinction predicted by HI alone. The dust associated with the molecular gas in the low velocity tail of Orion B can probably account for the extinction in excess of that expected from HI, suggesting that the three slightly reddened stars are located within the low velocity tail of Orion B which extends away from the main cloud toward the Sun. Two distances to Region 1 were estimated, a near distance of $\sim 420 \mathrm{pc}$ and a far distance of $\sim 520 \mathrm{pc}$. The distance to Region 2 was found to be $\sim 390 \mathrm{pc}$, though this estimate is rather uncertain as only a small number of suitable Hipparcos stars are found toward this part of Orion B.

A kinematic model where the stellar winds from the Ori OB $1 \mathrm{~b}$ subgroup have reached Orion B at its low-latitude and low-longitude corner is consistent with the observed distances. The gas at the interface has been compressed laterally and accelerated away from the Sun. This gas is located at the far distance. However, a small fraction of the gas has been accelerated toward the Sun. This gas, which does not appear to be fragmented (cf. the $2.3 \rightarrow 5.5 \mathrm{~km} \mathrm{~s}^{-1}$ channel map - Fig. 3), is estimated to have a mass of $1.2 \times 10^{4} M_{\odot}$, or about $15 \%$ of the total mass of the cloud.

\subsection{Orion East}

The contours in Fig. 7 are those of velocity-integrated $\mathrm{CO}$ in the cometary Orion East cloud, located toward the high latitude part of Orion B. The $\mathrm{H} \alpha$ emission from the region, shown in false colour in Fig. 7, indicates that the ionized gas is streaming around the cloud. The pressure of this gas may have triggered the recent star-formation that is suggested by the presence of at least five T Tauri stars (Herbig \& Rao 1972; Cohen \& Kuhi 1979) in the head of Orion East.

Orion East may be at a distance of only $120 \mathrm{pc}-$ significantly closer than Orion B and the only previous distance estimate of $500 \pm 140 \mathrm{pc}$ by Herbst (1982). The new estimate is based upon the parallaxes of only three Hipparcos stars, but since they all have small relative errors and could be assigned unambiguously, the new estimate is probably robust. In the kinematic model of the Orion-Eridanus bubble of Bally et al. (1998), Orion East has been accelerated toward the Sun and is on the near side of the bubble. The new distance estimate is in agreement with this model. It also agrees with estimates concerning the distance to the Orion-Eridanus bubble based upon X-ray intensity maps (Guo et al. 1995) which indicate that the near side of the bubble is at $159 \pm 16 \mathrm{pc}$ and the far side is at $226 \pm 24$ pc (Guo et al. 1995) or $\sim 400$ pc (Reynolds \& Ogden 1979).

Orion East is also noteworthy because it lies beyond the projected edge of the $\mathrm{H} \alpha$ emission associated with Barnard's Loop and the Orion-Eridanus bubble. Bally et al. (1998) argued that the Orion-Eridanus ionization boundary had receded, 


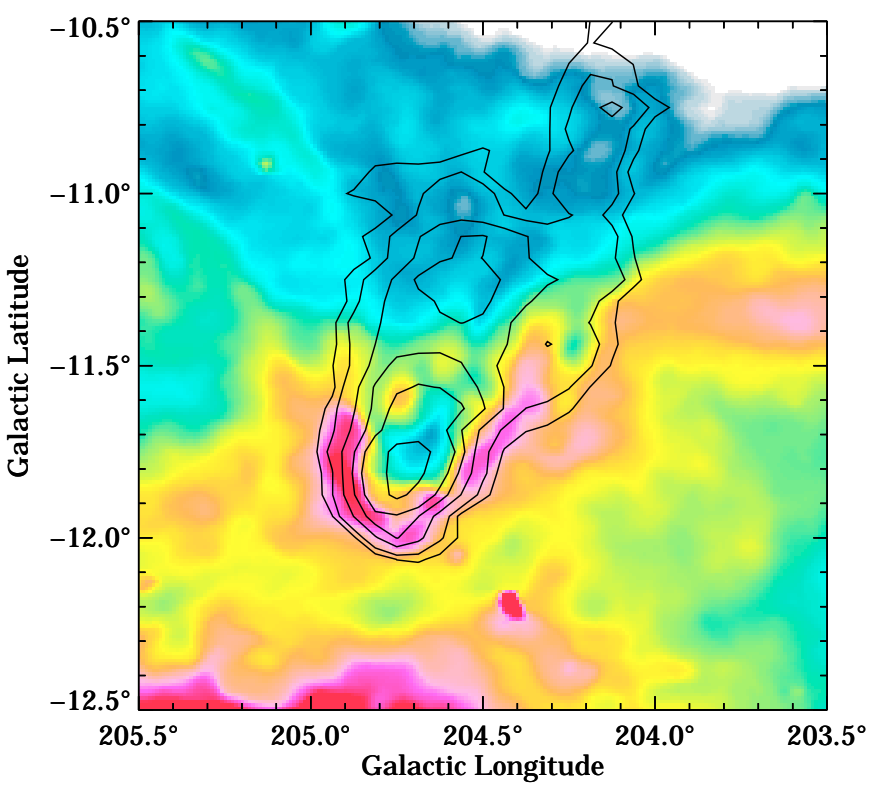

$40 \quad 6080100120$

$\mathrm{H} \alpha$ Intensity (Rayleighs)

Fig. 7. Ionized gas around Orion East (L 1622). The colour field represents the smoothed continuum subtracted $\mathrm{H} \alpha$ emission mapped by the Southern H $\alpha$ Sky Survey (SHASSA, Gaustad et al. 2001). Velocityintegrated $\mathrm{CO}$ emission from the globules is represented by the superimposed contours.

and that Barnard's Loop (and its faint $\mathrm{H} \alpha$ extensions into Eridanus) delineate the current ionization boundary of the Orion-Eridanus bubble, but in the past the influence of the OB association must have extended beyond this boundary in order to shape Orion East and the L 1617 globules into their present cometary form. However, Fig. 7 shows that ionized gas continues to stream around Orion East, and so a more likely explanation is that Barnard's Loop traces the ionization front of the near side of the Orion-Eridanus bubble, which is much closer to the Sun than the Orion-Monoceros molecular clouds.

\subsection{VDB 49: The Scissors}

At $l=202^{\circ}, b=-14^{\circ}$ is an unusual molecular cloud that we have dubbed the Scissors. The cloud covers an area of $\sim 2.2 \mathrm{deg}^{2}$, which at a distance of $\sim 150 \mathrm{pc}$ is equivalent to $\sim 15 \mathrm{pc}^{2}$, and it has a mass of $\sim 140 M_{\odot}$. The distinctive two limb structure and unusual kinematics of the cloud are summarized in Fig. 8, which shows the weighted mean velocity field of the cloud as a colour field with contours representing the velocity-integrated $\mathrm{CO}$ emission superimposed. The solid lines mark the positions and orientations of the two position-velocity slices, one along each limb, that are presented in Fig. 9.

The Scissors appears to be a large cometary cloud with a single head that points toward the centre of the OB $1 \mathrm{~b}$ subgroup and a forked tail that extends toward the Galactic plane. However, the two branches of the tail have very different kinematics as shown by the position-velocity slices, Fig. 9. The lower limb (slice 2) has a steep, smooth velocity gradient along

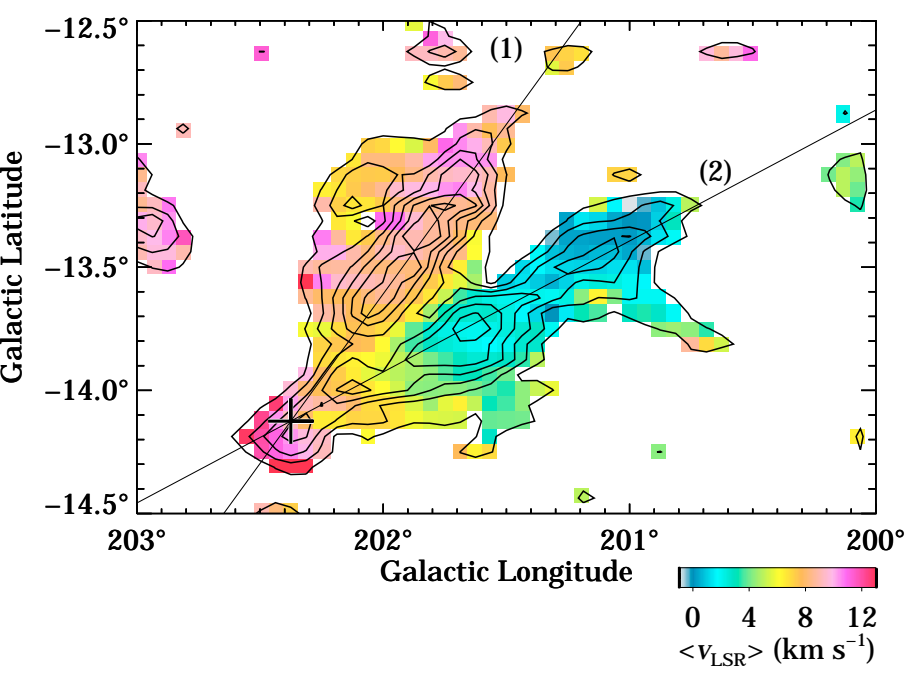

Fig. 8. The Scissors. The greyscale represents the emission weighted mean velocity and the contours the velocity-integrated $\mathrm{CO}$ intensity. The integration range is $-1<v_{\mathrm{LSR}}<16 \mathrm{~km} \mathrm{~s}^{-1}$ and the contours are at $1.8,3.6,5.4,7.2,9.0,10.8$ and $14.4 \mathrm{~K} \mathrm{~km} \mathrm{~s}^{-1}$. The two solid lines mark the positions of the position-velocity slices in Fig. 9. The cross marks the position of zero offset in these slices.
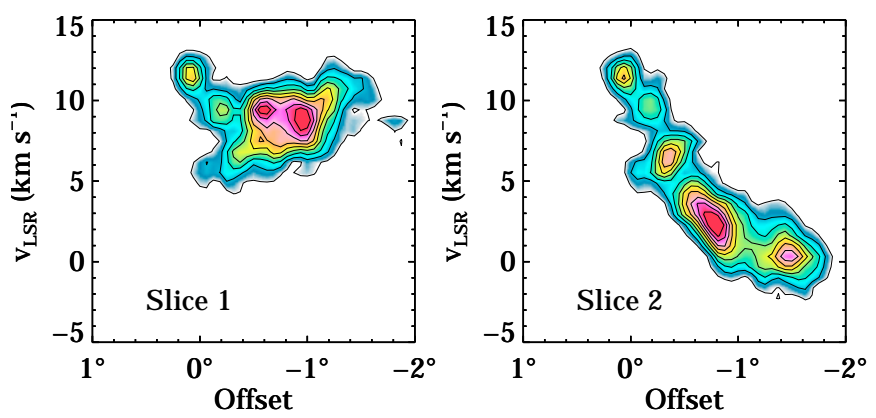

Fig. 9. Position-velocity slices through the Scissors. The orientation of the slices and the zero offset position are shown in Fig. 8. Each slice is integrated over a 0.375 slice. The contours in both plots start at $0.1 \mathrm{~K}$ arcdeg with subsequent steps at each additional $0.1 \mathrm{~K}$ arcdeg.

its entire length. This gradient is largely absent in the upper limb (slice 1). The apparent velocity discontinuity at the position where the two limbs intersect suggests that the Scissors are two separate clouds along the same line of sight. However, the probability of the chance alignment of two similarly shaped and oriented clouds $14^{\circ}$ from the Galactic plane is small.

It is not possible to determine the distance to either branch via the constraining star method as there are no Hipparcos stars that can be reliably assigned to the foreground of the Scissors. However, there are two stars, one for each branch, that can be assigned to the background and these indicate that neither branch of the Scissors is further away than $\sim 300 \mathrm{pc}$. We tentatively adopt a distance of $\sim 150 \mathrm{pc}$ to both. It is noted that the Scissors does not display a strong correlation between $\mathrm{H} \alpha$ emission and $W_{\mathrm{CO}}$ comparable to that of the nearby cloud Orion-East, which has a similar position close to Barnard's Loop and a more secure distance determination. 

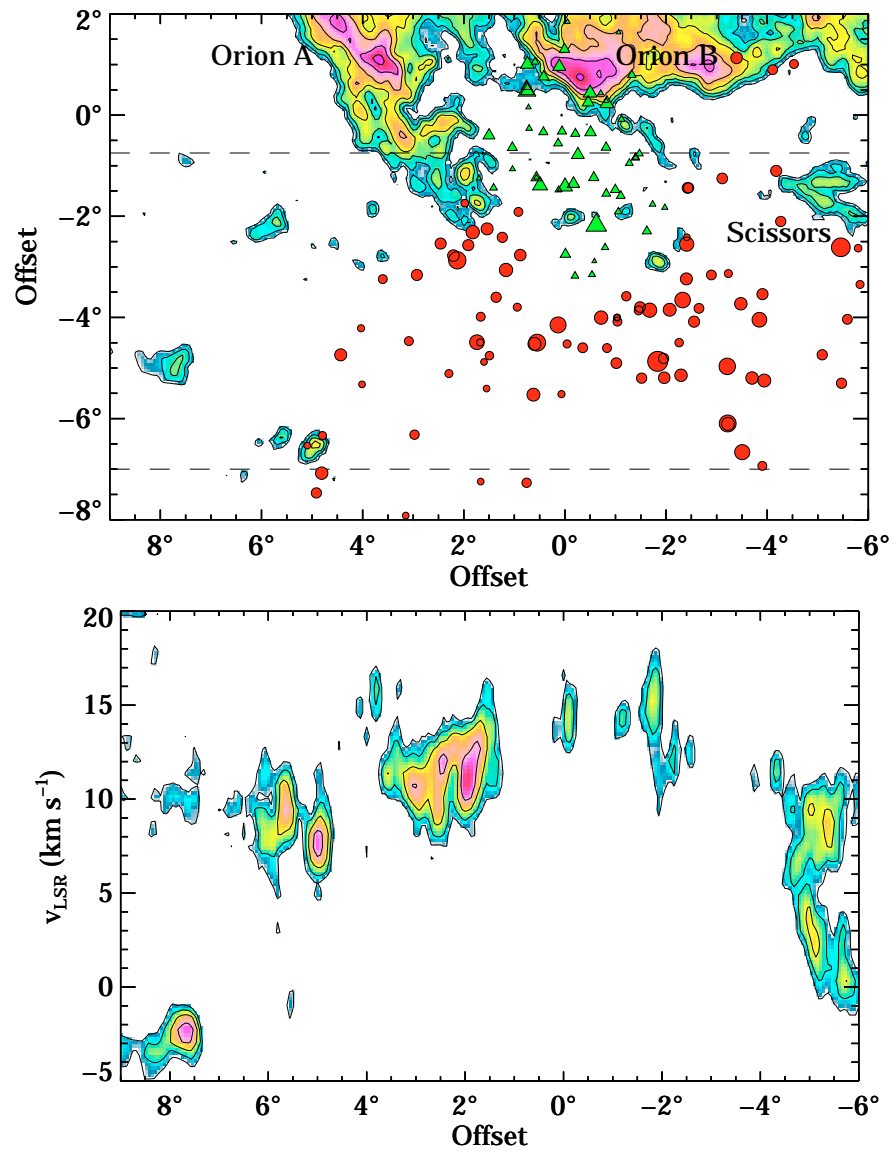

Fig. 10. Position-velocity slice through Ori OB 1 a. a) $W_{\text {CO }}$ map rotated $55^{\circ}$ clockwise about the position $l=206^{\circ}, b=-17^{\circ}$. The positions of $\mathrm{O}$ and $\mathrm{B}$ stars from the Ori 1a and Ori 1b subgroups are plotted with the same symbols as Fig. 1. The two horizontal dashed lines delineate the position-velocity slice. b) Position-velocity map for the Ori OB 1a region. The logarithmic contours begin at $-0.6(\sim 2 \sigma)$ and then proceed in steps of 0.3 .

A possible explanation for the unusual velocity structure of the Scissors is suggested by an examination of the kinematics of the surrounding gas. Figure 10 presents a positionvelocity map for a wide $\left(\sim 6^{\circ}\right)$ strip which includes the Scissors, the low longitude tip of the Orion A cloud and a number of small cometary clouds. Panel (a) shows the velocity-integrated $\mathrm{CO}$ map rotated $55^{\circ}$ clockwise about the position $l=206^{\circ}$, $b=-17^{\circ}$. The integration range for the position-velocity plot is shown by the two horizontal dashed lines. The positions of OB stars from the Ori OB $1 \mathrm{a}$ and $1 \mathrm{~b}$ subgroups (Brown et al $1995)$ are plotted with the same symbols as in Fig. 1 . The $W_{\mathrm{CO}}$ map is presented in this way so that a direct comparison with the position-velocity plot, panel (b), can be made. The kinematics of these clouds indicate that the Scissors forms part of a semi-circular structure that is consistent with expansion about the Orion OB 1a subgroup. The radius of the ring is $\sim 7^{\circ}$, which corresponds to a radius of $\sim 50 \mathrm{pc}$ at a distance of $\sim 400 \mathrm{pc}$. An expansion velocity of $8 \mathrm{~km} \mathrm{~s}^{-1}$ indicates that the ring is $\sim 6 \mathrm{Myr}$ old, approximately half the age of the OB 1a subgroup (Blaauw et al. 1991).

In this model of an expanding ring the high velocity $\left(\sim 12 \mathrm{~km} \mathrm{~s}^{-1}\right)$ clouds, characterized by the tip of Orion A, represent the most distant part of the ring and the low velocity gas $\left(\sim-1 \mathrm{~km} \mathrm{~s}^{-1}\right)$ in the Scissors and the globule at $x=8^{\circ}$, $y=-5^{\circ}$ in Fig. 10a represents the closest part of the ring. This is consistent with the model used to explain the kinematics of Orion East, but the actual distances derived using the constraining star technique indicate that the distance between the nearest and furthest part of the ring is $\sim 300 \mathrm{pc}$. The distance estimates may be in error, as they are based upon only a small number of stars, but it is also possible that the ring is older than $6 \mathrm{Myr}$ and that because of its orientation its actual size is underestimated.

\subsection{NGC 2149: A giant expanding ring?}

Between the Mon R2 cloud and the high longitude end of the Orion A cloud are a group of molecular clouds associated with the reflection nebula NGC 2149 (van den Bergh 1966). These clouds are at a significantly higher velocity $\left(\sim 12 \mathrm{~km} \mathrm{~s}^{-1}\right)$ than those in Orion $\mathrm{A}\left(\sim 5 \mathrm{~km} \mathrm{~s}^{-1}\right)$. In most projections these clouds appear to be unconnected. However, a three dimensional display of the data with velocity as a third dimension indicates that the clouds in this region may be connected to form a ring. This structure is on a much larger scale than the expanding shells located within Orion A.

The structure of the CO emission from NGC 2149 was modelled as an expanding ring. Figure 11 presents the fit of the model to the observations. (a) is the observed peak velocity field of NGC 2149, the ellipse represents the position and orientation of the model ring. (b) is a position-velocity diagram along the projected major axis of the model ring. The two plots in Fig. 11 suggest that the model is a reasonable fit to the data.

The total kinetic energy of the ring is estimated to be $\frac{1}{2} M_{\text {ring }} v_{\exp }^{2}=3 \times 10^{48} \mathrm{erg}$, where it is assumed that all of the gas in the NGC 2149 region is expanding, i.e. $M_{\text {ring }} \equiv M_{\mathrm{NGC} 2149}=$ $12.3 \times 10^{3} M_{\odot}$. At a distance of $400 \mathrm{pc}$ the radius of the ring is $\approx 40 \mathrm{pc}$, which corresponds to an expansion age of $\sim 9 \mathrm{Myr}$. The size and energetics of the NGC 2149 ring are comparable to those of the $\lambda$-Orionis ring (Lang et al. 2000). However, unlike the $\lambda$-Orionis ring, the NGC 2149 ring is not associated with a region of excess $\mathrm{H} \alpha$ emission and there are no stars comparable to $\lambda$-Ori to drive its expansion. The expansion may therefore be the result of a supernova.

\subsection{Mon R2}

Mon R2 is a massive star-forming cloud between Orion A and the Galactic plane. Its distance is claimed to be approximately $800 \mathrm{pc}$, significantly greater than that of Orion A or Orion B, but this estimate is quite uncertain. The constraining star technique provides only very rough upper and lower limits for the distance to the cloud, placing it only in the range 600-1100 pc. This estimate is consistent with the photometric determinations of $830 \pm 50$ (Racine 1968; Herbst \& Racine 1976) and 950 pc (Racine \& van den Bergh 1970). Mon R2 subtends an area of $\sim 15.3 \mathrm{deg}^{2}\left(\sim 3000 \mathrm{pc}^{2}\right)$ and has a mass of $\sim 0.75 \times 10^{5} \mathrm{M}_{\odot}$. Both the size and mass are comparable to those of Orion A. It is also located at approximately the same distance below the Galactic plane, $\sim 170 \mathrm{pc}$, and it has a similar velocity gradient 

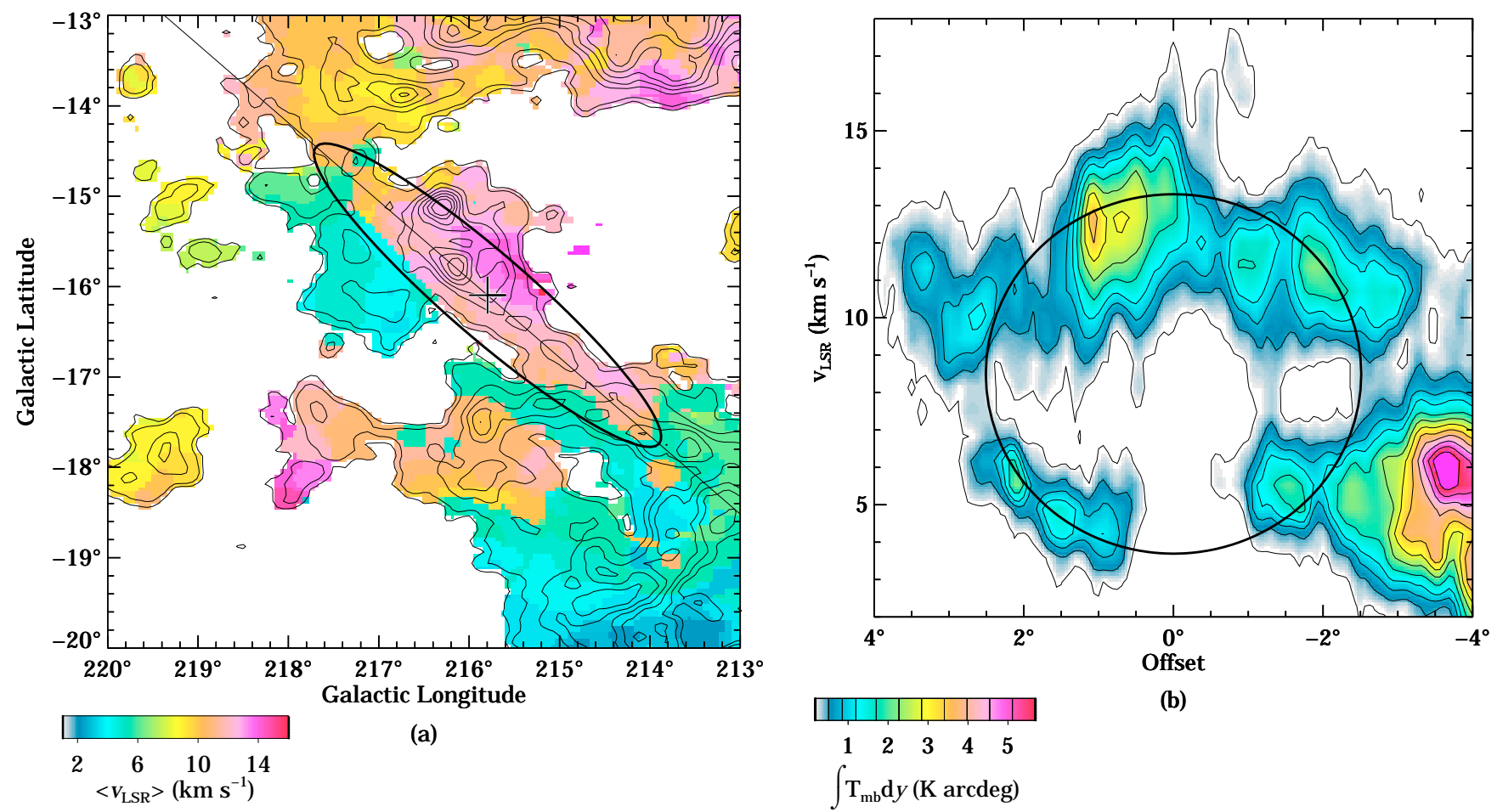

(b)

Fig. 11. a) Weighted mean velocity field of the NGC 2149 region. The integration range is $1<v_{\mathrm{LSR}}<16 \mathrm{~km} \mathrm{~s}^{-1}$ and the contours are at $1.7(3 \sigma)$, $2.8,7.3,10.1,13.0,15.8,18.6$ and $21.4 \mathrm{~K} \mathrm{~km} \mathrm{~s}^{-1}$. The solid ellipse marks the position of the expanding ring model fitted to the observations. The solid line shows the orientation of the position-velocity slice through the ring that is presented in $\mathbf{b}$ ), and the cross marks the location of the projected centre of the ellipse which is also the zero offset position. The position-velocity slice presented in $\mathbf{b}$ ) was integrated over $1^{\circ} .25$, the maximum extent of the projected ellipse. The contour levels start at 0.2 with subsequent levels at $0.5,0.9,1.2,1.7,2.3,2.8,3.4,4.0,4.5,5.1$ and $5.7 \mathrm{~K}$ arcdeg.

that runs roughly parallel to the Galactic plane and in the opposite direction to that expected from Galactic rotation (Hughes \& Baines 1985).

Mon R2 was first mapped in CO by Kutner \& Tucker (1975), then by Maddalena et al. (1986) and, at higher resolution, by Xie \& Goldsmith (1994) with the FCRAO 14 m telescope. Mon R2 has also been surveyed in ${ }^{13} \mathrm{CO}$ with the AT\&T Bell Laboratories $7 \mathrm{~m}$ telescope (Miesch \& Bally 1994). The high resolution surveys, and a smaller-scale multiline study of the high-density central core (Choi et al. 2000), have shown that Mon R2 has a unusual internal structure where most of the high density gas has been cleared from the central core and is constrained in a $\sim 30 \mathrm{pc}$ hemispherical shell. CO observations have also revealed the presence of two outflows; a large $200 M_{\odot}$ molecular outflow (Loren 1981; Wolf et al. 1990; Meyers-Rice \& Lada 1991) that originates from the main core and a much smaller outflow associated with the GGD 12-15 core (Little et al. 1990).

Figure 12a shows the integrated Mon R2 CO emission from the present survey. Mon R2 apparently contains two main structures: most of the emission comes from a pronounced spur that extends from the central core toward higher latitudes and the Galactic plane, or from the low-latitude side of the cloud that forms the roughly hemispherical shell (most clearly seen above the $16 \mathrm{~K} \mathrm{~km} \mathrm{~s}^{-1}$ contour) identified by Choi et al. (2000). Extending along the spur are three discrete knots of emission; the largest one, which is also the one closest to the main core, contains the GGD 12-15 core and associated outflow.

\subsubsection{The Mon R2 shell}

Xie \& Goldsmith (1994) reported that a large fraction of the molecular gas in Mon R2 is confined to a large $(\sim 30 \mathrm{pc})$ hemispherical shell, the projected centre of which is close to the NGC 2182 reflection nebula. This shell is obvious in the two CO position-velocity slices in Fig. 13, because they have been integrated only over the position of the core and not the whole cloud. The slices go through the position of the main outflow $(l=213 \circ 7, b=-12.6)$ and are orientated as shown by the solid lines in Fig. 12a. In Fig. 13 the main lobe of the outflow appears as a distinctive spike. Slice 1 runs roughly perpendicular to the major axis of the outflow, along the boundary between the low and high emission sides of Mon R2. Figure 13a is dominated by the outflow spike but the semi-circular velocity structure that characterizes the Mon R2 shell is also apparent. Slice 2 runs along the major axis of the outflow, as reported by Meyers-Rice \& Lada (1991); the associated position-velocity plot is shown in Fig. 13b. In addition to the outflow spike there is a large velocity gradient along the slice which characterizes the Mon R2 shell. The direction of the velocity gradient is not oriented in the same direction as the outflow, which accelerates gas at negative offsets toward lower velocities. This suggests 


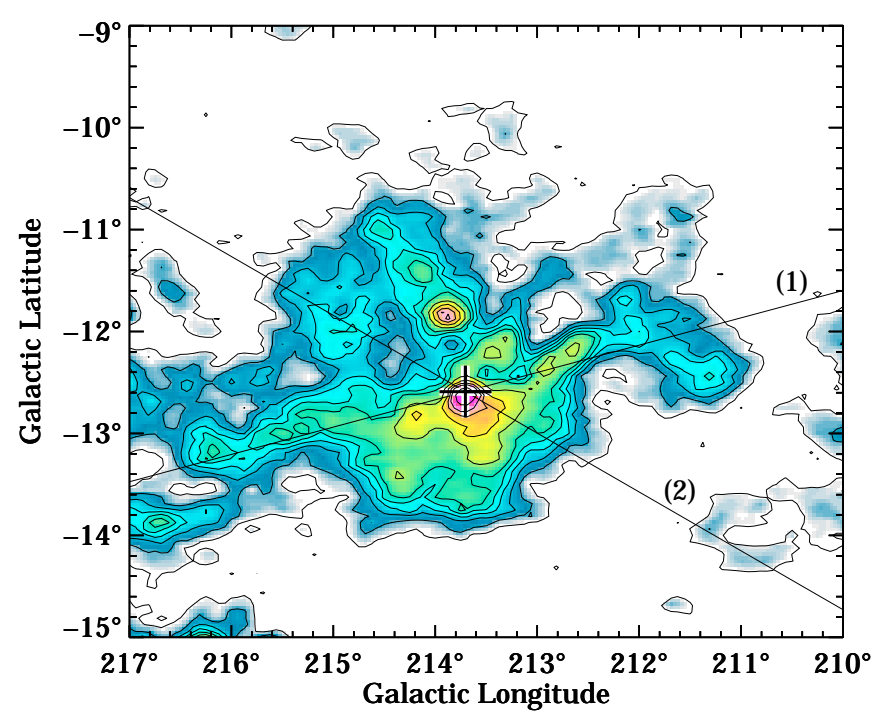

(a)

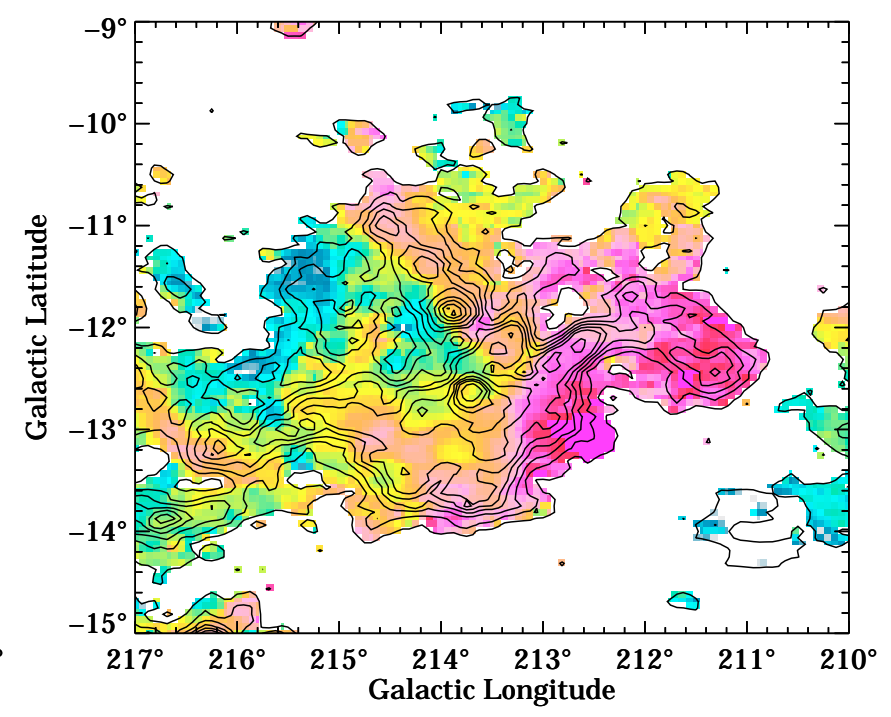

(b)

$$
\begin{aligned}
& 5 \quad 15 \quad 25 \quad 35 \quad 45 \\
& \int T_{m b} d v\left(K_{k m ~ s}{ }^{-1}\right)
\end{aligned}
$$

Fig. 12. Mon R2 in CO. a) $W_{\mathrm{CO}}$ map of Mon R2. The integration range is $5<v_{\mathrm{LSR}}<20 \mathrm{~km} \mathrm{~s}^{-1}$ and the contours are at $1.7(3 \sigma), 4.7$, 7.6, 10.5, 13.4, 16.3, 22.1, 27.9, 33.7, 39.6 and $51.2 \mathrm{~K} \mathrm{~km} \mathrm{~s}^{-1}$. The two solid lines mark the position velocity slices in Fig. 13. The cross at $l=213.71, b=-12.59$ marks the position of the Mon R2 outflow. b) Mon R2 emission-weighted mean velocity field with $W_{\mathrm{CO}}$ contours superimposed.

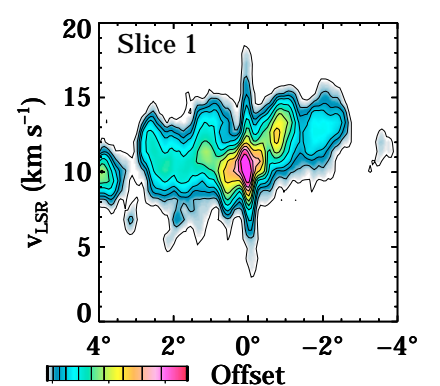

$0.2 \quad 1.42 .6$

$\int \mathrm{T}_{\mathrm{mb}} \mathrm{dy}(\mathrm{K}$ arcdeg)

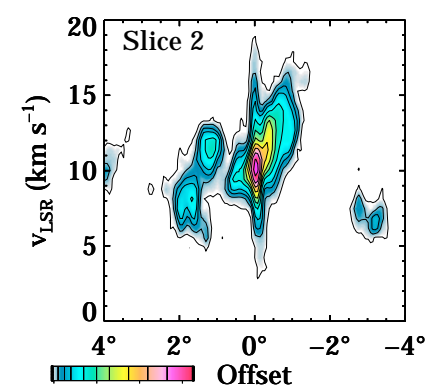

0.10 .81 .52 .2 (b)

Fig. 13. Two position-velocity slices through Mon R2. The positions and orientations of the slices are shown in Fig. 12a. Each slice was integrated over $\sim 0.25$. Contour levels are indicated by the colour bars.

that the outflow is not responsible for the shell. Furthermore, it is clear from Fig. 13 that the main core is a coherent part of the velocity structure of the shell, and is therefore likely to be located within it.

\subsubsection{The Mon R2 spur}

Xie \& Goldsmith (1994) suggested that the sharp edge of the spur was evidence of recent formation, plausibly by shock compression. The corrugations that appear along the spur are also consistent with the kinematic instability of shock front development (Elmegreen \& Combes 1992). Since the main Mon R2 outflow at $l=213.7, b=-12^{\circ} .6$ is the most conspicuous energy source in the cloud, it is worth asking if the spur is related to it.

The spur originates from a position very close to the main Mon R2 outflow (marked as a cross in Fig. 12a), and it is conceivable that gas entrained by the outflow has been aligned along the spur by some mechanism. However, for any mechanism to be credible it must also explain two significant observations. First, the orientation of the outflow, as determined by Meyers-Rice \& Lada (1991), differs by $\sim 45^{\circ}$ from the orientation of the spur, and second, the base of the spur is at least 0.5 from the outflow. Jets driving smaller outflows have been observed to precess (e.g. HH 315 Arce \& Goodman 2002), and this mechanism could explain the observed differences between the orientation of the clumps and the outflow, and the offset between the base of the spur and the outflow. Nevertheless, the length of the spur $(\sim 30 \mathrm{pc})$ may be too large for this to be realistic. Wolf et al. (1990) estimated the age of the outflow to be $1.5 \times 10^{5} \mathrm{yr}$, so for the outflow to have swept the most distant clump out to $30 \mathrm{pc}$ the gas would have had to have a velocity of $300 \mathrm{~km} \mathrm{~s}^{-1}$. Although this is comparable to the speed of the jet, it is much faster than the speed of the gas entrained by the outflow and so it is therefore more likely that another, older, mechanism has aligned the spur.

The projected centre of the shell $\left(l=213^{\circ} .9, b=-12^{\circ} .2\right)$ is about halfway between the two dense cores (the main core and the GGD 12-15 core) that contain the two main Mon R2 outflows. When compared with the centroid of the Mon R2 shell the spur appears to be cometary, with a well defined rounded head centered on the GGD 12-15 core and a tail that extends toward low latitudes. Xie \& Goldsmith (1994) estimated the dynamical age of the shell to be $\sim 4 \times 10^{6} \mathrm{yr}$ and its total kinetic energy to be $5-20 \times 10^{48} \mathrm{erg}$. A similar amount of energy deposited over about the same length of time might produce the observed structure of the spur. 


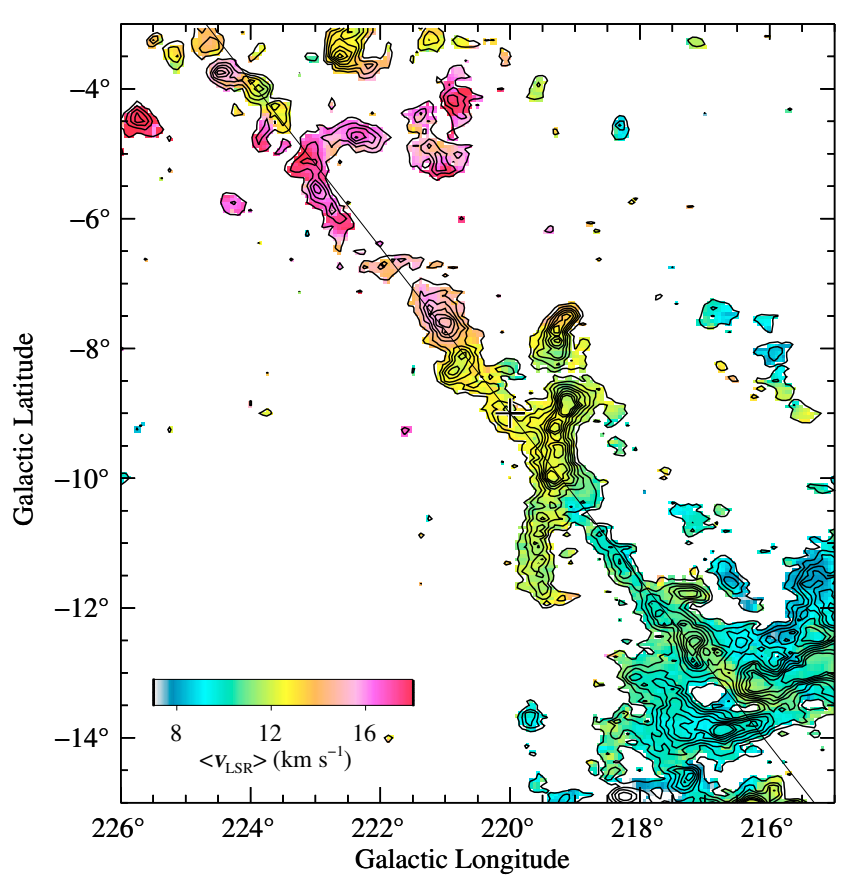

(a)

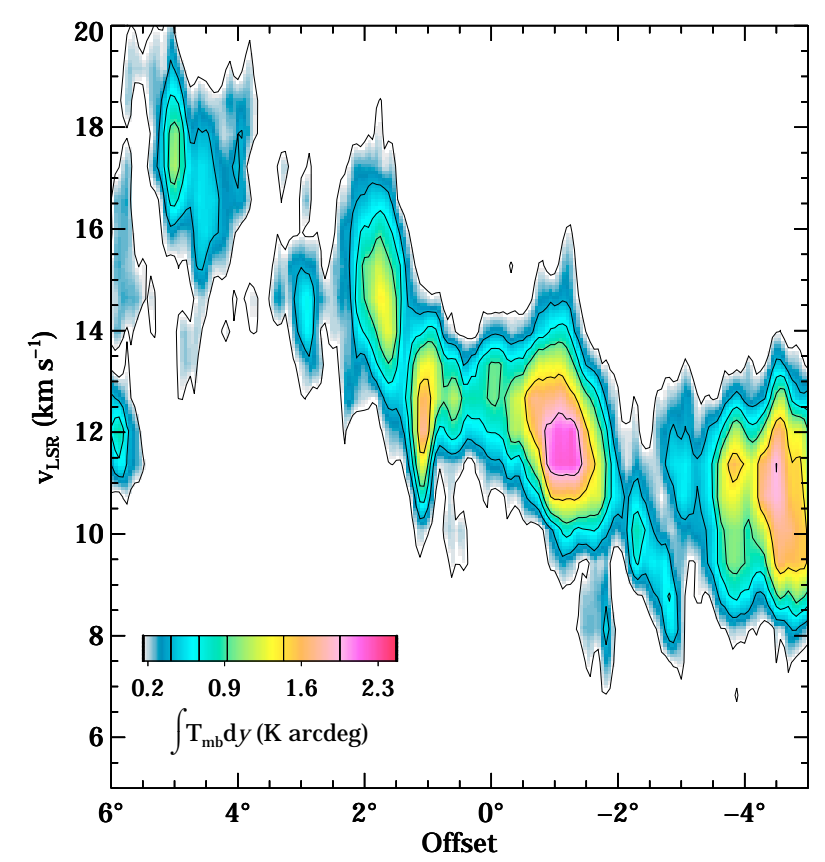

(b)

Fig. 14. a) Emission-weighted mean velocity map of the Southern Filament with $W_{\mathrm{CO}}$ contours superimposed. Contours start at $1.69 \mathrm{~K} \mathrm{~km} \mathrm{~s}^{-1}$ $(3 \sigma)$ with subsequent steps every $1.69 \mathrm{~K} \mathrm{~km} \mathrm{~s}^{-1}$. The solid line shows the orientation of the position-velocity slice presented in $\mathbf{b}$ ), with the cross marking the position of zero offset. b) Position-velocity plot for the Southern Filament. The data was integrated over a $\sim 1^{\circ}$ band centered on the filament. Contour levels are indicated by the colour bar.

\subsubsection{Star-formation in Mon R2}

Mon R2 is a very active region of star-formation with two main condensations centered on the positions of the largest bipolar outflows (Shimmins et al. 1966; Beckwith et al. 1976; Thronson et al. 1980; Cohen \& Schwartz 1980; Little et al. 1990). Herbst \& Racine (1976) suggested that there have been two distinct phases of star-formation in Mon R2, the first of which produced the many A- and B-type stars and associated reflection nebulosity in the cloud. This phase occurred at least $6 \times 10^{6} \mathrm{yr}$ ago, consistent with the dynamical age of the hemispherical shell (Xie \& Goldsmith 1994). The second period started $10^{4}-10^{5}$ yr ago (Beckwith et al. 1976; Loren 1977; Xie \& Goldsmith 1994) and it is continuing within the dense cores and shock compressed ridges that are associated with the bipolar outflows, compact $\mathrm{HII}$ regions, $\mathrm{H}_{2} \mathrm{O}$ masers and IRAS point sources of the cloud. The correlation between the location of star-formation in Mon R2 and the dense gas that was shockcompressed by the previous generation implies triggered starformation.

\subsection{The Southern Filament and the Crossbones}

Extending from close to the Mon R2 cloud to the Galactic plane is the Southern Filament, a remarkably straight and narrow molecular cloud, more than $10^{\circ}$ in length and generally less than $1^{\circ}$ wide. The filament has a relatively smooth gradient characterized by higher velocities at higher latitudes. The velocity gradient can be seen in the emission-weighted mean velocity map and position-velocity slice, Figs. 14a and b. The location and orientation of the position-velocity slices is shown by the solid line in Fig. 14a, and its nominal centre by the cross at $l=220^{\circ}, b=-9^{\circ}$.

Distances were determined for two parts of the Southern Filament, the regions above and below $b \sim-7^{\circ}$; both found to be at $\sim 460 \mathrm{pc}$. However, these estimates were based upon only three and five stars respectively and consequently have large relative errors (see Table 2). Despite the uncertainty in the distance estimate it seems more likely that the Southern Filament is related to the NGC 2149 ring and high longitude end of Orion A than Mon R2. However, there is not a clear boundary between the Southern Filament and Mon R2 in either projection or position-velocity space. Possible reasons for the kinematic similarity between the gases at different distances are explored in Sect. 5.

The distinctive Crossbones clouds, first identified by Maddalena et al. (1986), at $l \approx 219^{\circ}, b \approx-10^{\circ}$, may simply be the superposition of two clouds along the line of sight, but the similar kinematics of the two limbs suggests that this is unlikely. There is some evidence, presented in Fig. 15, that star-formation is currently taking place within the Crossbones, near the position where the two limbs intersect: (a), the $100 \mu \mathrm{m}$ infrared emission measured by IRAS, shows several knots of emission consistent with the presence of embedded young stellar objects (YSOs). Most promisingly the knot at the intersection of the two limbs, $l \approx 219^{\circ} .2, b \approx-9^{\circ} .9$ is coincident with the position of an apparent bipolar outflow suggested by the well defined spike in the velocity-latitude map in (c). 


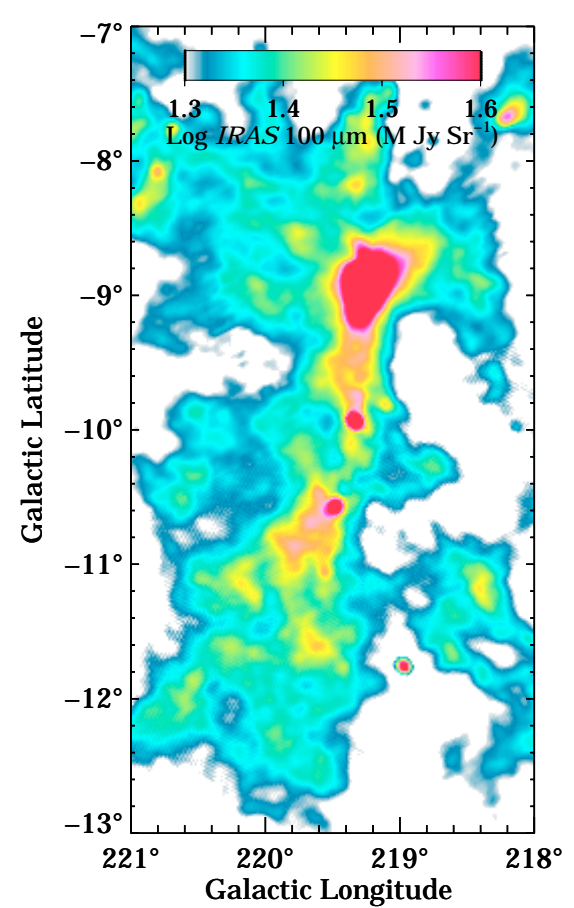

(a)

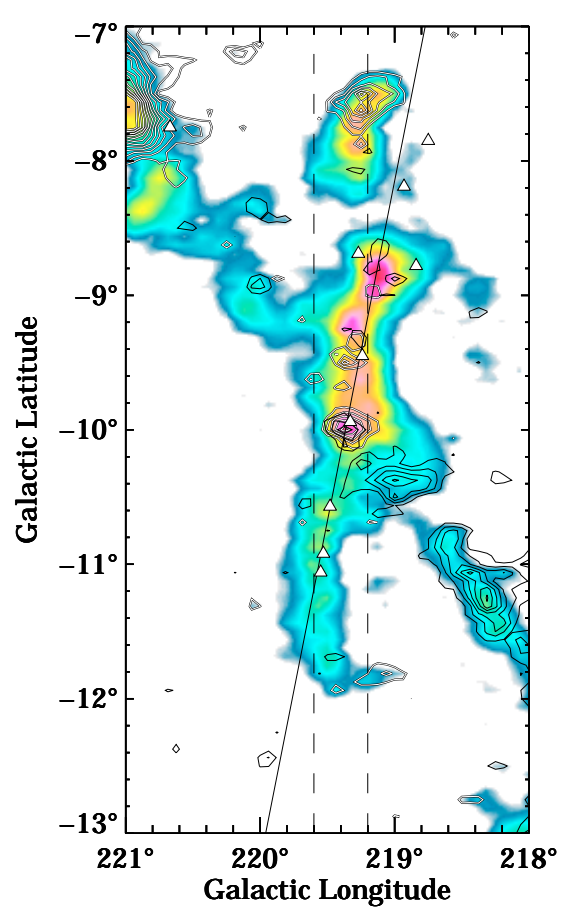

(b)

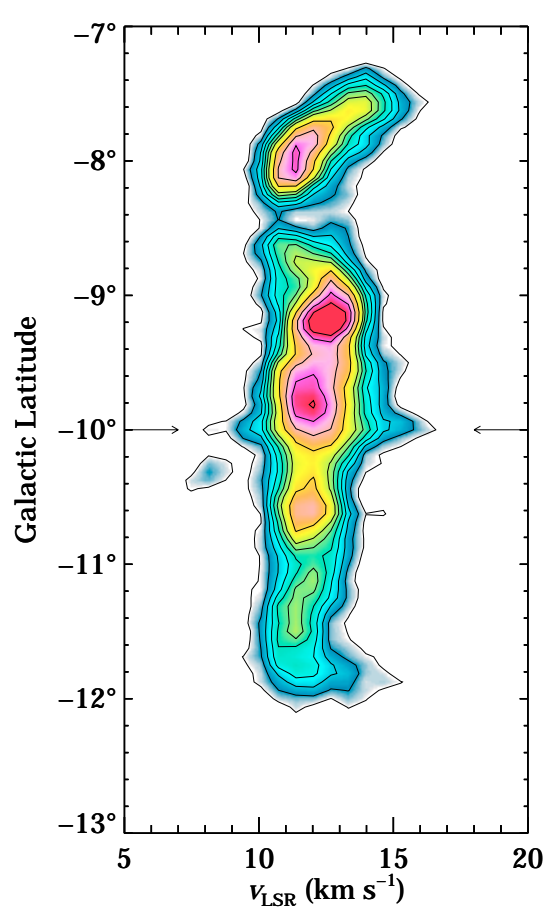

(c)

Fig. 15. a) Map of IRAS $100 \mu \mathrm{m}$ emission from the Crossbones. b) $W_{\mathrm{CO}}$ map of the Crossbones. The integration range is $6.0<v_{\mathrm{LSR}}<$ $18.0 \mathrm{~km} \mathrm{~s}^{-1}$. The white $\left(14.5<v_{\mathrm{LSR}}<18.0 \mathrm{~km} \mathrm{~s}^{-1}\right)$ and black $\left(7.0<v_{\mathrm{LSR}}<9.5 \mathrm{~km} \mathrm{~s}^{-1}\right)$ contours show the distribution of high and low velocity gas respectively. The two vertical dashed lines show the integration range for the velocity-latitude map presented in c). The crosses mark the positions of colour selected IRAS point sources that are potentially EYSOs. The solid line delineates the inferred major axis of the apparent bipolar outflow that is centered on $l=219^{\circ} .2, b=-10^{\circ} .0$. c) Velocity-latitude map of the Crossbones. The integration range is $219^{\circ} .2<l<219^{\circ} .6 \mathrm{~km} \mathrm{~s}^{-1}$ and the contours start at $0.1 \mathrm{~K}$ arcdeg with subsequent steps every additional $0.1 \mathrm{~K}$ arcdeg. The arrows at $b=-10^{\circ} .0$ mark the position of the velocity spike associated with the apparent outflow.

\subsection{The Northern Filament}

Figure $16 \mathrm{a}$ shows the $W_{\mathrm{CO}}$ map of a second long filament, comparable to the Southern Filament described above, that is located between the high-latitude end of the Orion B cloud and the Galactic plane. This cloud was first mapped in CO by Maddalena et al. (1986), who called it the Northern Filament. However, this name is slightly misleading because although the Northern Filament is similar in length and orientation to the Southern Filament, it is neither as narrow nor as straight. It is possible that this cloud would not have been described as a filament at all had it not been aligned parallel to the Southern Filament.

The distance to the Northern Filament is estimated as $\sim 390 \mathrm{pc}$ by the constraining star technique, the same as the distance to the high latitude part of Orion B. In addition to being approximately at the same distance, the Northern Filament and Orion B have very similar morphologies and kinematics along their adjacent edges, suggesting that they were once part of a coherent structure.

The Northern Filament is relatively large and massive: it subtends an area of $15.3 \mathrm{deg}^{2}$ on the sky and has a mass of $0.13 \times 10^{6} M_{\odot}$. Despite its size, the cloud is remarkably quiescent. Molecular clouds of this size typically have starformation efficiencies of a fraction of one percent, which corresponds here to $\approx 200$ young stars. There is nothing in the Northern Filament to indicate that so much star-formation is taking place: there are no T Tauri stars, HII regions, reflection nebulae, bipolar outflows or IRAS point sources with colours similar to those of EYSOs.

The kinematics of the Northern Filament, as summarized by the velocity-latitude diagram, Fig. 16b, are fairly unremarkable, which is not surprising considering its quiescence. Unlike the Southern Filament, there is no large scale velocity gradient, and the whole cloud is constrained to velocities close to $\sim 10 \mathrm{~km} \mathrm{~s}^{-1}$. Part of the reason why the velocity-latitude plot appears so smooth is because it has been integrated over a relatively large longitude range, $200^{\circ}<l<210^{\circ}$; narrower slices do reveal a more complex kinematic structure. In particular there are several positions where the small-scale velocity structure of the cloud matches that of Orion B at the same longitude.

\section{Large-scale structure of Orion-Monoceros}

The similarity between the Orion A and Mon R2 clouds has already been noted (Sect. 4.6). They are at approximately the same distance from the plane, they have about the same size and mass, and they both have large-scale velocity gradients in a direction opposite to that of Galactic rotation. Figure 17b is a position-velocity slice through the emission peaks of the Orion A and Mon R2 clouds. The orientation of the slice is delineated by the dashed line in Fig. 17a which shows a composite of two of the Orion-Monoceros channel maps (Fig. 3). 

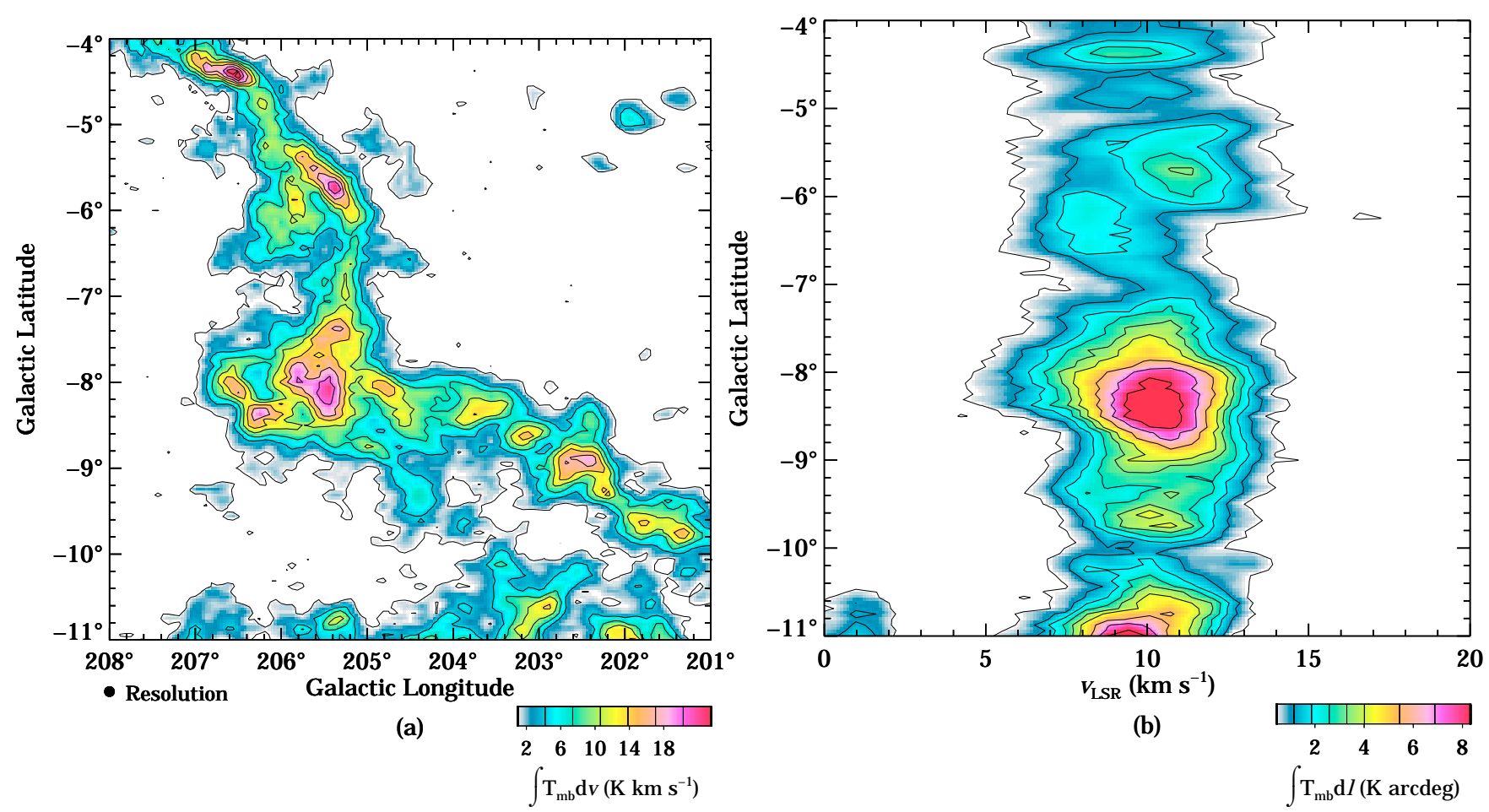

Fig. 16. a) $W_{\mathrm{CO}}$ map of the Northern Filament. The integration range is $4<v_{\mathrm{LSR}}<16 \mathrm{~km} \mathrm{~s}^{-1}$. The contours start at $0.97 \mathrm{~K} \mathrm{~km} \mathrm{~s}{ }^{-1}(3 \sigma)$ with subsequent steps every additional $3.22 \mathrm{~K} \mathrm{~km} \mathrm{~s}^{-1}(10 \sigma)$. b) Velocity-latitude map of the Northern Filament. The integration range is $201^{\circ}<l<$ $210^{\circ}$ and the contours are at $0.4,1.2,1.9,2.6,3.3,4.0,5.4,6.9$ and $8.3 \mathrm{~K} \mathrm{~km} \mathrm{~s}^{-1}$.

The map is presented in this way to emphasize that the slice is parallel to the Northern and Southern Filaments and that it passes along emission spurs in both Orion A and Mon R2 that are also parallel to the filaments. The morphological and kinematic similarity between Orion A and Mon R2 is striking. Both clouds are home to giant molecular outflows, as characterised by the emission spikes at offsets of $-5^{\circ} .5$ and $3^{\circ}$, both have lobes of high-velocity gas on their low Galactic latitude sides and both have spurs of emission that extend from their central core toward the Galactic plane and are parallel to the Southern Filament.

Mon R2 is more that $\sim 300 \mathrm{pc}$ further away than Orion A and has not usually been considered to be part of the Orion complex. However, when confronted by the numerous similarities between Orion A and Mon R2 it is difficult to avoid speculating that these clouds, and by extension all the clouds in the region, share a common origin.

Heiles (1998) proposed that the formation of OrionMonoceros was associated with the expansion of the Vela supershell (GSH238+00+09), which may have originated from the cluster Collinder 121 (Cr 121) located near $l \sim 235^{\circ}$, $b \sim-10^{\circ}, v_{\mathrm{LSR}}=17 \pm 4 \mathrm{~km} \mathrm{~s}^{-1}$ (Blaauw 1991), and at a distance of $546 \pm 30 \mathrm{pc}$ (Hoogerwerf et al. 1997). The Vela supershell encloses an enormous irregular bubble in the region where it has blown out of the Galactic plane, one side of which runs approximately parallel to the Southern Filament, before becoming more spread out at lower latitudes.

Heiles (1998) claimed that the Vela supershell follows the expansion law $R \propto t^{0.31}$, meaning that the walls of the supershell had travelled $\sim 80 \%$ of the way to their present location at half the age. Thus the Vela supershell would have overrun the position of the Orion OB association at about the time that the OB 1a subgroup was formed.

Bally et al. (1998) proposed that a collision of the Vela supershell with a fossil remnant of the Lindblad Ring may have played a role in triggering the formation of the proto OrionMonoceros complex. They suggest that the ISM was first compressed when it was swept up by the Lindblad Ring supershell; as the expansion of this superbubble slowed the dense gas in the shell was compressed further by the impact of the Vela supershell, enhancing the tendency of the gas to collapse via gravitational instability and form stars.

The structure of Orion-Monoceros close to the Orion OB association is now dominated by the local effects of stellar winds, supernova explosions and the expansion of the OrionEridanus bubble, but much of the underlying large-scale structure is consistent with the formation sequence described above. In particular the alignment of the Southern Filament and the Orion A and Mon R2 spurs with the wall of the Vela superbubble does not appear to be purely coincidental.

\section{Summary and conclusions}

This paper presents the results of a new CO survey of the Orion-Monoceros complex carried out with the HarvardSmithsonian $1.2 \mathrm{~m}$ telescope. The new observations are more sensitive and have better sampling than the only previous large survey of the region (Maddalena et al. 1986). Distances to the majority of the clouds in Orion-Monoceros are determined using the constraining star technique and the Hipparcos catalogue. The new distances aided the three dimensional analysis 


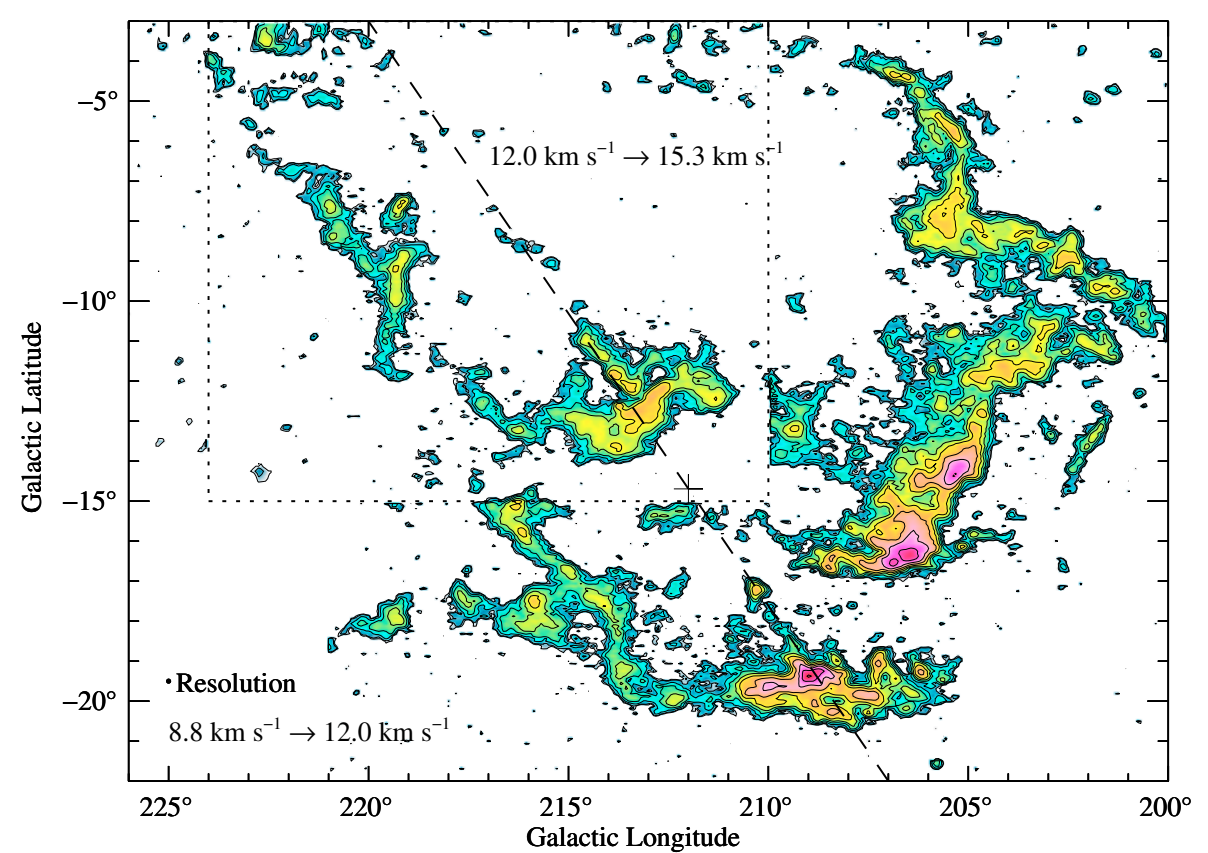

(a)

$$
\begin{aligned}
& \begin{array}{lllll}
0.0 & 0.5 & 1.0 & 1.5 & 2.0
\end{array} \\
& \log \int \mathrm{T}_{\mathrm{mb}} \mathrm{d} v\left(\mathrm{~K} \mathrm{~km} \mathrm{~s}^{-1}\right)
\end{aligned}
$$

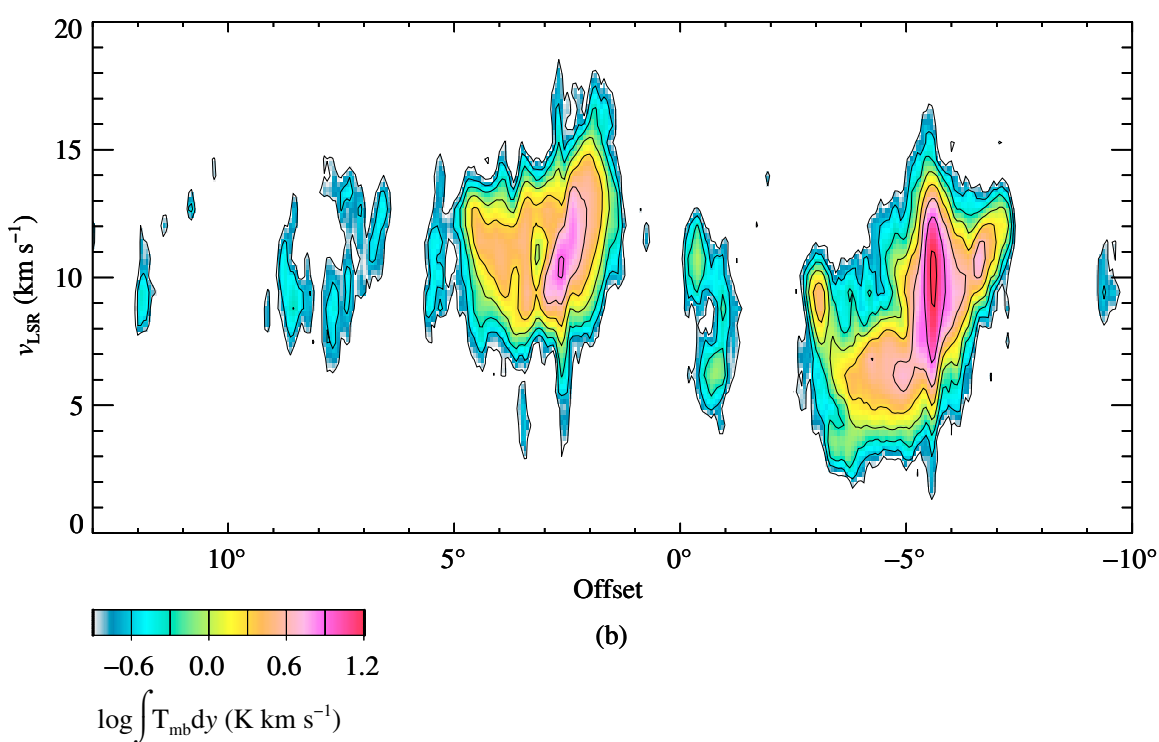

Fig. 17. a) Composite $W_{\mathrm{CO}}$ map of Orion-Monoceros. The area within the box marked by the dotted line has been integrated over the range $12.0<v_{\mathrm{LSR}}<15.3 \mathrm{~km} \mathrm{~s}^{-1}$, with the remainder of the map integrated over the range $8.8<v_{\mathrm{LSR}}<12.0 \mathrm{~km} \mathrm{~s}^{-1}$. The contours are the same as the channel maps in Fig. 3. The dashed line delineates the position-velocity slice presented in $\mathbf{b})$ and the cross marks the position of zero offset. b) Position-velocity slice through Orion A and Mon R2. The contours start at $-0.9(\sim 3 \sigma)$ and go up in steps of 0.3 (log $K \operatorname{arcdeg})$.

of the molecular gas and it relationship to the other phases of the ISM. The main conclusions of this analysis are as follows:

1. Stellar winds and ionizing radiation from the massive stars of the Ori OB $1 \mathrm{~b}$ and $\mathrm{OB} 1 \mathrm{~d}$ subgroups are responsible for changes to the morphology and kinematics of the molecular gas in Orion A and Orion B today.
2. The kinematics and three dimensional structure of Orion $\mathrm{A}$ and Orion B are consistent with stellar wind driven compression centered on Ori OB $1 \mathrm{~b}$. Knots of compressed gas are coincident with the most active star-forming regions in Orion A and Orion B, suggesting that triggering may have occurred. 
3. The Orion-Eridanus superbubble of hot $\mathrm{H} \alpha$ emitting gas inflated by the Orion $\mathrm{OB}$ association and delineated in projection by Barnard's loop has overrun many small clumps of dense gas. These globules have cometary shapes caused by their interaction with the expanding bubble, and many display signs of enhanced (triggered) star-formation in their heads. However, only one globule, Orion-East, displays a fairly unambiguous connection to the ionizing radiation of Barnard's Loop, and this globule is significantly closer than the main molecular clouds of the complex. Therefore, we conclude that Barnard's Loop delineates the closest part of the Orion-Eridanus bubble and not the part adjacent to Orion-Monoceros.

4. The most distant cloud in the complex, Mon R2, is quite similar to the large molecular cloud associated with the Orion nebula that is significantly closer. Both clouds have spurs of emission that are aligned with the Southern Filament (and the Vela supershell). These features suggest that the clouds of the region share a common origin.

5. The new CO survey supports the proposals by Bally et al. (1998) and Heiles (1998) that the formation of the OrionMonoceros complex was triggered by the passage of the Vela supershell and possibly its interaction with a preexisting supershell associated with the Lindblad Ring.

Acknowledgements. Part of this work was supported by the Particle Physics and Astronomy Research Council (PPARC) grant number PPA/S/S/1998/02675. The authors are grateful to E.S. Palmer for skillfully maintaining the $1.2 \mathrm{~m}$ telescope, and to Jodie Dalton and Adam Kampff for their help with the observations.

\section{References}

Anthony-Twarog, B. J. 1982, AJ, 87, 1213

Arce, H. A., \& Goodman, A. A. 2002, ApJ, 575, 911

Bally, J., Stark, A. A., Wilson, R. W., \& Langer, W. D. 1987, ApJ, 312, L45

Beckwith, S., Evans, N. J., Becklin, E. E., \& Neugebauer, G. 1976, ApJ, 208, 390

Blaauw, A. 1991, in The Physics of Star Formation and Early Stellar Evolution, ed. C. J. Lada, \& N. D. Kylafis (Dordrecht: Kluwer), 342,125

Brown, A. G. A., de Geus, E. J., \& de Zeeuw, P. T. 1994, A\&A, 289, 101

Brown, A. G. A., Hartmann, D., \& Burton, W. B. 1995, A\&A, 300, 903

Cambrésy, L. 1999, A\&A, 345, 965

Castets, A., Duvert, G., Dutrey, A., et al. 1990, A\&A, 234, 469

Chin, G. 1977, Ph.D. Thesis, Columbia University

Choi, M., Evans, N. J., Tafalla, M., \& Bachiller, R. 2000, ApJ, 538, 738

Cohen, M., \& Kuhi, L. V. 1979, ApJS, 41, 743

Cohen, M., \& Schwartz, R. D. 1980, MNRAS, 191, 165

Cowie, L. L., Songaila, A., \& York, D. G. 1979, ApJ, 230, 469
Dame, T. M., Koper, E., Israel, F. P., \& Thaddeus, P. 1993, ApJ, 418, 730

Davis, J. H., Loren, R. B., \& vanden Bout, P. A. 1973, BAAS, 5, 420

Elmegreen, B. G., \& Combes, F. 1992, A\&A, 259, 232

Elmegreen, B. G., \& Lada, C. J. 1977, ApJ, 214, 725

Genzel, R., Reid, M. J., Moran, J. M., \& Downes, D. 1981, ApJ, 244, 884

Guo, Z., Burrows, D. N., Sanders, W. T., Snowden, S. L., \& Penprase, B. E. 1995, ApJ, 453, 256

Heiles, C. 1998, ApJ, 498, 689

Heiles, C., Haffner, L. M., \& Reynolds, R. J. 1999, in New Perspectives on the Interstellar Medium, ASP Conf. Ser., 168, 211

Herbig, G. H., \& Kameswara Rao, N. 1972, ApJ, 174, 401

Herbst, W., Holtzman, J. A., \& Phelps, B. E. 1982, AJ, 87, 1710

Herbst, W., \& Racine, R. 1976, AJ, 81, 840

Heyer, M. H., Morgan, J., Schloerb, F. P., Snell, R. L., \& Goldsmith, P. F. 1992, ApJ, 395, L99

Hillenbrand, L. A. 1997, AJ, 113, 1733

Hoogerwerf, R., de Bruijne, J. H. J., Brown, A. G. A., et al. 1997, in Hipparcos-Venice '97 (The Netherlands: Noordwijk), ESA SP 402, 571

Hughes, V. A., \& Baines, J. G. N. 1985, ApJ, 289, 238

Kramer, C., Stutzki, J., \& Winnewisser, G. 1996, A\&A, 307, 915

Kutner, M. L. 1978, Astrophys. Lett., 19, 81

Kutner, M. L., \& Tucker, K. D. 1975, ApJ, 199, 79

Kutner, M. L., Tucker, K. D., Chin, G., \& Thaddeus, P. 1977, ApJ, 215,521

Lang, W. J. 1997, Ph.D. Thesis, Bristol University

Lang, W. J., Masheder, M. R. W., Dame, T. M., \& Thaddeus, P. 2000, A\&A, 357, 1001

Little, L. T., Heaton, B. D., \& Dent, W. R. F. 1990, A\&A, 232, 173

Loren, R. B. 1977, ApJ, 215, 129

Loren, R. B., Mundy, L., \& Erickson, N. R. 1981, ApJ, 250, 573

Lynds, B. T. 1962, ApJS, 7, 1

Maddalena, R. J., Moscowitz, J., Thaddeus, P., \& Morris, M. 1986, ApJ, 303, 375

Meyers-Rice, B. A., \& Lada, C. J. 1991, ApJ, 368, 445

Miesch, M. S., \& Bally, J. 1994, ApJ, 429, 645

Nagahama, T., Mizuno, A., Ogawa, H., \& Fukui, Y. 1998, AJ, 116, 336

Pan, S. K., Feldman, M. J., Kerr, A. R., \& Timbie, P. 1983, Appl. Phys. Lett., 43, 786

Penzias, A. A., \& Burrus, C. A. 1973, ARA\&A, 11, 51

Racine, R. 1968, AJ, 73, 233

Racine, R., \& van den Bergh, S. 1970, in The Spiral Structure of our Galaxy, IAU Symp., 38, 219

Reynolds, R. J., \& Ogden, P. M. 1979, ApJ, 229, 942

Reynolds, R. J., Tufte, S. L., Haffner, L. M., Jaehnig, K., \& Percival, J. W. 1998, PASA, 15, 14

Sakamoto, S., Hayashi, M., Hasegawa, T., Handa, T., \& Oka, T. 1994, ApJ, 425, 641

Shimmins, A. J., Clarke, M. E., \& Ekers, R. D. 1966, ApJ, 19, 649

Thronson, H. A., Gatley, I., Harvey, P. M., Sellgren, K., \& Werner, M. W. 1980, ApJ, 237, 66

van den Bergh, S. 1966, AJ, 71, 990

Warren, W. H., \& Hesser, J. E. 1977, ApJS, 34, 207

Wilson, B. A. 2002, Ph.D. Thesis, Bristol University

Wilson, R. W., Jefferts, K. B., \& Penzias, A. A. 1970, ApJ, 161, L43

Wolf, G. A., Lada, C. J., \& Bally, J. 1990, AJ, 100, 1892

Xie, T., \& Goldsmith, P. F. 1994, ApJ, 430, 252 